\title{
A Functional Integral Representation for Many Boson Systems I: The Partition Function
}

\author{
Tadeusz Balaban \\ Department of Mathematics \\ Rutgers, The State University of New Jersey \\ 110 Frelinghuysen $\mathrm{Rd}$ \\ Piscataway, NJ 08854-8019 \\ tbalaban@math.rutgers.edu \\ Joel Feldman* \\ Department of Mathematics \\ University of British Columbia \\ Vancouver, B.C. \\ CANADA V6T $1 \mathrm{Z2}$ \\ feldman@math.ubc.ca \\ http://www.math.ubc.ca/ feldman/ \\ Horst Knörrer, Eugene Trubowitz \\ Mathematik \\ ETH-Zentrum \\ CH-8092 Zürich \\ SWITZERLAND \\ knoerrer@math.ethz.ch, trub@math.ethz.ch \\ http://www.math.ethz.ch/ knoerrer/
}

\begin{abstract}
We derive a functional integral representation for the partition function of a many Boson system for which the configuration space consists of finitely many points.
\end{abstract}

* Research supported in part by the Natural Sciences and Engineering Research Council of Canada and the Forschungsinstitut für Mathematik, ETH Zürich. 


\section{Introduction}

We are developing a set of tools and techniques for analyzing the large distance/infrared behaviour of a system of identical bosons, as the temperature tends to zero.

The total energy of the many boson systems considered in this paper has two sources. First, each particle in the system has a kinetic energy. We shall denote the corresponding quantum mechanical observable by $h$. The most common is $-\frac{1}{2 m} \Delta$, but, in this paper, we allow any positive operator. Second, the particles interact with each other through a twobody potential, $v(\mathbf{x}, \mathbf{y})$. For stability, $v$ is required to be repulsive. We assume that the system is in thermodynamic equilibrium and that expectations of observables are given by the grand canonical ensemble at temperature $T=\frac{1}{k \beta}>0$ and chemical potential $\mu$.

Functional integrals are an important source of intuition about the behaviour of quantum mechanical systems. They are also an important rigorous technical tool in the analysis of, for example, Euclidean quantum field theories. In this paper and its companion [II], we derive rigorous functional integral representations for the partition function and thermodynamic correlation functions of a many boson system.

There are many possible applications of our functional integral representations. However, we are motivated by the following potential specific application. One may speculate (in agreement with the standard picture of condensed matter physics) that, at temperature zero and infinite volume, a weakly coupled, three dimensional many boson system will undergo a phase transition at some critical chemical potential $\mu_{*}$.

- For $\mu<\mu_{*}$, the system is in a massive phase. That is, all correlation functions decay exponentially fast at large separation. The expected value of the field $\phi(\mathbf{x})=\left\langle a^{\dagger}(\mathbf{x})\right\rangle$, where $a^{\dagger}(\mathbf{x})$ is the particle creation operator at $\mathbf{x}$, is zero.

○ For $\mu>\mu_{*}$, number symmetry is broken. In this phase, correlation functions fail to decay exponentially due to the presence of extended, collective excitations (the massless Goldstone bosons). The expected value of the field is nonzero. The presence of such anomolous nonzero amplitudes is used as a general criterion for a condensed quantum fluid.

The intuition behind this phase transition is easily obtained by using a formal coherent state functional integral [NO, (2.66)] to express the grand canonical partition function as

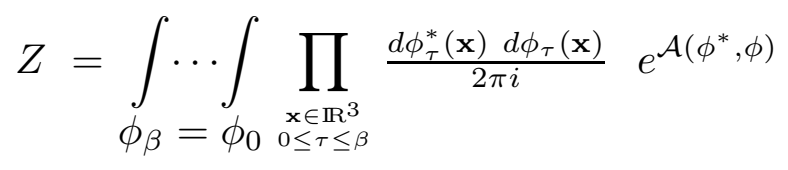

where

$$
\mathcal{A}\left(\phi^{*}, \phi\right)=\int_{0}^{\beta} d \tau \int_{\mathbb{R}^{3}} d^{3} \mathbf{x} \quad \phi_{\tau}^{*}(\mathbf{x}) \frac{\partial}{\partial \tau} \phi_{\tau}(\mathbf{x})-\int_{0}^{\beta} d \tau K\left(\phi_{\tau}^{*}, \phi_{\tau}\right)
$$


and

$$
\begin{aligned}
K\left(\alpha^{*}, \phi\right)= & \iint d \mathbf{x} d \mathbf{y} \alpha(\mathbf{x})^{*} \mathrm{~h}(\mathbf{x}, \mathbf{y}) \phi(\mathbf{y})-\mu \int d \mathbf{x} \alpha(\mathbf{x})^{*} \phi(\mathbf{x}) \\
& +\frac{1}{2} \iint d \mathbf{x} d \mathbf{y} \alpha(\mathbf{x})^{*} \alpha(\mathbf{y})^{*} v(\mathbf{x}, \mathbf{y}) \phi(\mathbf{x}) \phi(\mathbf{y})
\end{aligned}
$$

Here $\mathrm{h}(\mathbf{x}, \mathbf{y})$ is the kernel of the kinetic energy operator.

In the mean field approximation, that is, when $\phi_{\tau}(\mathbf{x})$ is independent of $\tau$ and $\mathbf{x}$, the action $\mathcal{A}\left(\phi^{*}, \phi\right)$ is minus the integral over $\tau$ and $\mathbf{x}$ of the

$$
\text { "naive effective potential" }=\frac{1}{2} \hat{v}(0)|\phi|^{4}-\mu|\phi|^{2}
$$

where $\hat{v}(0)=\int d \mathbf{y} v(\mathbf{x}, \mathbf{y})$. We have assumed that $v(\mathbf{x}, \mathbf{y})$ is translation invariant and that $\mathrm{h}$ annihilates constants. The minimum of the naive effective potential is

$\circ$ nondegenerate at the point $\phi=0$ when $\mu<0$

and

$\circ$ degenerate along the circle $|\phi|=\sqrt{\frac{\mu}{\hat{v}(0)}}$ when $\mu>0$.

It is therefore reasonable that an attempt to rigorously justify the phase transition in the chemical potential discussed above would begin with the derivation of rigorous functional integral representations of the thermodynamic correlation functions in which the effective potential appears explicitly. We do so in this paper and the companion paper [II].

It is common practice in condensed matter physics, to discretize space, because the overall energy scale is low. On physical grounds, this does not affect the long range behaviour of the system. For this paper, space is an arbitrary, but fixed, finite set $X$, that we may imagine is a subset of a lattice. The second quantized Hamiltonian $\mathrm{H}$ and the number operator $N$ act on the infinite dimensional Fock space

$$
\mathcal{F}=\bigoplus_{n=0}^{\infty}\left(\mathbb{C}^{|X|^{n}} / S_{n}\right)
$$

The long distance behavior of the system is revealed in the thermodynamic limits of grand canonical correlation functions, such as derivatives of the partition function

$$
Z=\operatorname{Tr}_{\mathcal{F}} e^{-\beta(\mathrm{H}-\mu N)}
$$

To implement the thermodynamic limit, one would take the usual family of finite spaces

$$
X_{L}=\left\{x \in \mathbb{Z}^{3}|| x_{i} \mid<L, i=1,2,3\right\}
$$

and send $L$ to infinity. We shall not do so in this paper.

Our first result (Theorem III.13), stated somewhat informally, is the representation

$$
\operatorname{Tr} e^{-\beta(\mathrm{H}-\mu N)}=\lim _{p \rightarrow \infty} \int \prod_{\tau \in \mathcal{T}_{p}} d \mu_{\mathrm{R}(p)}\left(\phi_{\tau}^{*}, \phi_{\tau}\right) e^{\mathcal{F}\left(\frac{\beta}{p}, \phi^{*}, \phi\right)}
$$


for the finite volume grand canonical partition function. Similar representations for general correlation functions are derived in [II]. Here, for each natural number $p$, the discrete time interval $\mathcal{T}_{p}$ is given by

$$
\mathcal{T}_{p}=\left\{\tau=q \frac{\beta}{p} \mid q=1, \cdots, p\right\}
$$

For each point $(\mathbf{x}, \tau)$ in the discrete space-time $X \times \mathcal{T}_{p}$, we have introduced the complex variable $\phi(\tau, \mathbf{x})=\phi_{\tau}(\mathbf{x})$. For each $r>0$, the measure

$$
d \mu_{r}\left(\phi^{*}, \phi\right)=\prod_{\mathbf{x} \in X}\left[\frac{d \phi^{*}(\mathbf{x}) \wedge d \phi(\mathbf{x})}{2 \pi \imath} \chi_{r}(|\phi(\mathbf{x})|)\right]
$$

where, $\chi_{r}$ is the characteristic function of the closed interval $[0, r]$. The sequence $\mathrm{R}(p)>0$ in (I.2) tends to infinity at an appropriate rate as $p \rightarrow \infty$. The "action" $\mathcal{F}\left(\varepsilon, \phi^{*}, \phi\right)$, with $\varepsilon=\frac{\beta}{p}$, is given by

$$
\begin{aligned}
\mathcal{F}\left(\varepsilon, \phi^{*}, \phi\right)= & \iint d \tau d \mathbf{x} \phi_{\tau}^{*}(\mathbf{x})\left(\partial^{\varepsilon} \phi_{\tau}\right)(\mathbf{x})-\iint d \tau d \mathbf{x} \phi_{\tau}^{*}(\mathbf{x})\left(\mathrm{h} \phi_{\tau}\right)(\mathbf{x})+\mu \iint d \tau d \mathbf{x} \phi_{\tau}(\mathbf{x})^{*} \phi_{\tau}(\mathbf{x}) \\
& -\frac{1}{2} \iiint d \tau d \mathbf{x} d \mathbf{y} \phi_{\tau}(\mathbf{x})^{*} \phi_{\tau}(\mathbf{x}) v(\mathbf{x}, \mathbf{y}) \phi_{\tau}(\mathbf{y})^{*} \phi_{\tau}(\mathbf{y})
\end{aligned}
$$

where

$$
\begin{aligned}
\iint d \tau d \mathbf{x} \psi(\tau, \mathbf{x}) & =\varepsilon \sum_{\tau \in \mathcal{T}_{p}} \sum_{\mathbf{x} \in X} \psi(\tau, \mathbf{x}) \\
\iiint d \tau d \mathbf{x} d \mathbf{y} \psi(\tau, \mathbf{x}, \mathbf{y}) & =\varepsilon \sum_{\tau \in \mathcal{T}_{p}} \sum_{\mathbf{x} \in X} \sum_{\mathbf{y} \in X} \psi(\tau, \mathbf{x}, \mathbf{y})
\end{aligned}
$$

and the difference operator $\partial^{\varepsilon}$ acts by

$$
\partial^{\varepsilon} \phi(\tau, \mathbf{x})=\varepsilon^{-1}(\phi(\tau+\varepsilon, \mathbf{x})-\phi(\tau, \mathbf{x}))
$$

In (I.2), fields $\phi_{\tau}$ with $\tau \notin \mathcal{T}_{p}$ are determined by the periodicity condition $\phi_{\tau}=\phi_{\tau-\beta}$. It is easy to check that the representation (I.2) generates the usual formal graphical perturbation series. the identity

In the physics literature, coherent $\operatorname{states}^{(1)}|\phi\rangle, \phi \in \mathbb{C}^{X}$, the formal resolution of

$$
\mathbb{1}=\int \prod_{\mathbf{x} \in X}\left[\frac{d \phi^{*}(\mathbf{x}) d \phi(\mathbf{x})}{2 \pi \imath} e^{-\left|\phi_{\tau}(\mathbf{x})\right|^{2}}\right]|\phi\rangle\langle\phi|
$$

and the formal trace formula

$$
\operatorname{Tr} B=\int \prod_{\mathbf{x} \in X}\left[\frac{d \phi^{*}(\mathbf{x}) d \phi(\mathbf{x})}{2 \pi \imath} e^{-\left|\phi_{\tau}(\mathbf{x})\right|^{2}}\right]\langle\phi|B| \phi\rangle
$$

(1) We are using coherent states normalized so that $\langle\alpha \mid \gamma\rangle=e^{\int d \mathbf{y} \overline{\alpha(\mathbf{y})} \gamma(\mathbf{y})}$. We systematically use the convention that $\int d \mathbf{x} f(\mathbf{x})=\sum_{\mathbf{x} \in X} f(\mathbf{x})$. 
are used to justify (I.1) as follows. Formally,

$$
\begin{aligned}
& \operatorname{Tr} e^{-\beta(\mathrm{H}-\mu N)}=\lim _{p \rightarrow \infty} \operatorname{Tr} e^{-\frac{\beta}{p}(\mathrm{H}-\mu N)} \mathbb{1} e^{-\frac{\beta}{p}(\mathrm{H}-\mu N)} \mathbb{1} \cdots \mathbb{1} e^{-\frac{\beta}{p}(\mathrm{H}-\mu N)} \\
& =\lim _{p \rightarrow \infty} \int \prod_{\substack{\mathbf{x} \in X \\
\tau \in \mathcal{T}_{p} \backslash\{\beta\}}}\left[\frac{d \phi_{\tau}^{*}(\mathbf{x}) d \phi_{\tau}(\mathbf{x})}{2 \pi \imath} e^{-\left|\phi_{\tau}(\mathbf{x})\right|^{2}}\right] \operatorname{Tr}\left[\prod_{\tau \in \mathcal{T}_{p} \backslash\{\beta\}} e^{-\frac{\beta}{p}(\mathrm{H}-\mu N)}\left|\phi_{\tau}\right\rangle\left\langle\phi_{\tau}\right|\right] e^{-\frac{\beta}{p}(\mathrm{H}-\mu N)} \\
& =\lim _{p \rightarrow \infty} \prod_{\substack{\mathbf{x} \in X \\
\tau \in \mathcal{T}_{p}}}\left[\int \frac{d \phi_{\tau}^{*}(\mathbf{x}) d \phi_{\tau}(\mathbf{x})}{2 \pi \imath} e^{-\left|\phi_{\tau}(\mathbf{x})\right|^{2}}\right] \prod_{\tau \in \mathcal{T}_{p}}\left\langle\phi_{\tau}\left|e^{-\frac{\beta}{p}(\mathrm{H}-\mu N)}\right| \phi_{\tau+\frac{\beta}{p}}\right\rangle
\end{aligned}
$$

Then, one argues [NO, (2.59)] that

$$
\begin{aligned}
\left\langle\alpha\left|e^{-\varepsilon(\mathrm{H}-\mu N)}\right| \phi\right\rangle & =\left\langle\alpha\left|: e^{-\varepsilon(\mathrm{H}-\mu N)}:\right| \phi\right\rangle+O\left(\varepsilon^{2}\right) \\
& =\exp \left\{\int d \mathbf{y} \alpha(\mathbf{y})^{*} \phi(\mathbf{y})-\varepsilon K\left(\alpha^{*}, \phi\right)+O\left(\varepsilon^{2}\right)\right\}
\end{aligned}
$$

where : $\cdot$ : denotes Wick ordering. If $\varepsilon=\frac{\beta}{p}$, one observes that there are $O(p)$ error terms of order $O\left(\varepsilon^{2}\right)=O\left(\frac{1}{p^{2}}\right)$. "Off the cuff", the error terms do not contribute when $p \rightarrow \infty$ and consequently,

$$
\operatorname{Tr} e^{-\beta(\mathrm{H}-\mu N)}=\lim _{p \rightarrow \infty} \prod_{\substack{\mathbf{x} \in X \\ \tau \in \mathcal{T}_{p}}}\left[\int \frac{d \phi_{\tau}^{*}(\mathbf{x}) d \phi_{\tau}(\mathbf{x})}{2 \pi \imath}\right] \prod_{\tau \in \mathcal{T}_{p}} e^{\int d \mathbf{y} \phi_{\tau}(\mathbf{y})^{*}\left[\phi_{\tau+\beta / p}(\mathbf{y})-\phi_{\tau}(\mathbf{y})\right]-\frac{\beta}{p} K\left(\phi_{\tau}^{*}, \phi_{\tau+\beta / p}\right)}
$$

It is the purpose of this paper, and its companion, to make this all precise.

In $\S I I$, we review the basic formalism of bosonic quantum statistical mechanics and the formalism of coherent states in the context of a finite configuration space $X$. In particular, we prove, in Theorem II.26, a rigorous version of the formal resolution of the identity (I.3) and we prove, in Proposition II.28, that the trace formula (I.4) applies rigorously to a certain class of operators. In this way, we obtain a rigorous variant of (I.5). See Theorem III.1.

It is by no means clear that dropping, "off the cuff", the $O(p)$ error terms of order $O\left(\varepsilon^{2}\right)$ is justified, because the error terms are unbounded functions of the fields $\phi_{\tau}$. We circumvent this part of the formal argument by directly constructing the logarithm $F\left(\varepsilon, \alpha^{*}, \phi\right)=\ln \left\langle\alpha\left|e^{-\varepsilon K}\right| \phi\right\rangle$, at least for $\alpha$ and $\phi$ not too large. See Proposition III.6. To this end, we derive and then solve an evolution equation in $\varepsilon$ for $F\left(\varepsilon, \alpha^{*}, \phi\right)$. It follows that

$$
F\left(\varepsilon, \alpha^{*}, \phi\right)=\int_{X} d \mathbf{x} \alpha(\mathbf{x})^{*} \phi(\mathbf{x})-\varepsilon K\left(\alpha^{*}, \phi\right)+O\left(\varepsilon^{2}\right)
$$

We then show, in Theorem III.13, that the "matrix element" $\left\langle\alpha\left|e^{-\varepsilon(\mathrm{H}-\mu N)}\right| \phi\right\rangle$ can be replaced by $e^{\int d \mathbf{y} \alpha(\mathbf{y})^{*} \phi(\mathbf{y})-\varepsilon K\left(\alpha^{*}, \phi\right)}$ in the formula for $\operatorname{Tr} e^{-\beta(\mathrm{H}-\mu N)}$ of Theorem III.1, provided the integration radius $\mathrm{R}(p)$ of (I.2) is chosen appropriately. 
In the physics literature, one simply "evaluates" the limit

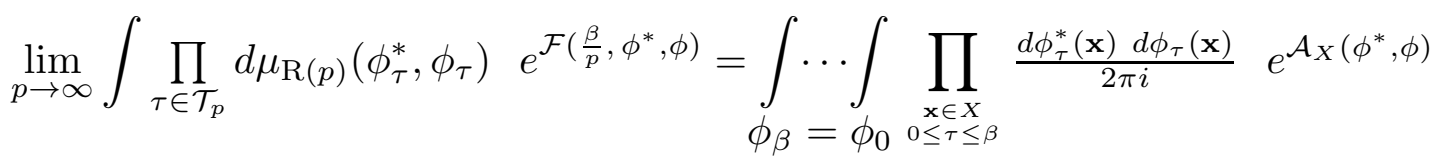

where

$$
\begin{aligned}
\mathcal{A}_{X}\left(\phi^{*}, \phi\right)=\int_{0}^{\beta} d \tau & \int_{X} d \mathbf{x}\left\{\phi_{\tau}^{*}(\mathbf{x}) \frac{\partial}{\partial \tau} \phi_{\tau}(\mathbf{x})-\phi_{\tau}^{*}(\mathbf{x})(\mathrm{h}-\mu) \phi_{\tau}(\mathbf{x})\right\} \\
& -\frac{1}{2} \int_{0}^{\beta} d \tau \iint_{X^{2}} d \mathbf{x} d \mathbf{y} \phi_{\tau}(\mathbf{x})^{*} \phi_{\tau}(\mathbf{y})^{*} v(\mathbf{x}, \mathbf{y}) \phi_{\tau}(\mathbf{x}) \phi_{\tau}(\mathbf{y})
\end{aligned}
$$

The first impulse of a mathematical physicist determined to ascribe a rigorous meaning to this formal functional integral representation for the partition function, would be to construct a "complex Gaussian measure" $d \mu_{C}$, with covariance

$$
C=\left(-\frac{\partial}{\partial \tau}+(\mathrm{h}-\mu)\right)^{-1}
$$

out of the formal measure

$$
\exp \left\{\int_{0}^{\beta} d \tau \int_{X} d \mathbf{x}\left\{\phi_{\tau}^{*}(\mathbf{x}) \frac{\partial}{\partial \tau} \phi_{\tau}(\mathbf{x})-\phi_{\tau}^{*}(\mathbf{x})(\mathrm{h}-\mu) \phi_{\tau}(\mathbf{x})\right\}\right\} \prod_{\substack{\mathbf{x} \in X \\ 0 \leq \tau \leq \beta}} \frac{d \phi_{\tau}^{*}(\mathbf{x}) d \phi_{\tau}(\mathbf{x})}{2 \pi i}
$$

Normally, one starts by defining the integral of any polynomial in the complex fields $\phi_{\tau}^{*}(\mathbf{x}), \phi_{\tau}(\mathbf{x}), \tau \in[0, \beta), \mathbf{x} \in X$, against $d \mu_{C}$ as cumulants of "matrix elements" of the covariance $C$. Then one constructs the characteristic function of $d \mu_{C}$, as a limit of integrals of polynomials, and the corresponding measure. However, the explicit calculations in Appendix A, modelled on those of Cameron $[\mathrm{C}]$, show that the purely imaginary term

$$
\int_{0}^{\beta} d \tau \int_{X} d \mathbf{x} \phi_{\tau}^{*}(\mathbf{x}) \frac{\partial}{\partial \tau} \phi_{\tau}(\mathbf{x})
$$

in the exponential generates oscillations that are so severe that there is no complex Gaussian measure.

To work with the ultraviolet limit

$$
\lim _{p \rightarrow \infty} \int \prod_{\tau \in \mathcal{T}_{p}} d \mu_{\mathrm{R}(p)}\left(\phi_{\tau}^{*}, \phi_{\tau}\right) e^{\mathcal{F}\left(\frac{\beta}{p}, \phi^{*}, \phi\right)}
$$

to, for example, construct the thermodynamic limit and justify the phase transition in the chemical potential, one must exploit the cancellations arising from the oscillations generated by the purely imaginary term in the action. 
One methodology for the explicit control of cancellations of this kind, when the coupling constant $\lambda=\hat{v}(0)$ is small, is known as "multiscale analysis". In the present case "scales" refer to blocks of frequencies in space $\mathbf{x}$ and inverse temperature $\tau$. There are infinitely many scales. Cancellations are implemented at each scale.

Typically, the total contribution of "large fields", for example field configurations with $\phi_{\tau}(\mathbf{x})$ or appropriate derivatives large, is smaller than any power of $\lambda$, reminiscent of large deviations in probability theory. This can be proven without attention to cancellations. On the other hand, oscillations are fully exploited in the complementary "small field regions" by high dimensional steepest descent calculations around the complex critical points of the effective actions. In the end the functional integral becomes an infinite sum over small and large field regions. The physicists formal functional integral is morally "the dominant term". 


\section{Finite Systems of Bosons}

In this section we carefully review the basic formalism of bosonic quantum statistical mechanics and introduce the notation that we will systematically use.

\section{Fock Space}

Fix a finite set $X$.

\section{Definition II.1}

(i) Let $n \in \mathbb{N} \cup\{0\}$. The action $\pi \cdot f$ of a permutation $\pi \in S_{n}$ on $f$ in $L^{2}\left(X^{n}\right)$ is given by

$$
\pi \cdot f\left(\mathbf{x}_{1}, \cdots, \mathbf{x}_{n}\right)=f\left(\mathbf{x}_{\pi^{-1}(1)}, \ldots, \mathbf{x}_{\pi^{-1}(n)}\right)
$$

The bosonic $n$-particle space

$$
\mathcal{B}_{n}(X)=\left\{f \in L^{2}\left(X^{n}\right) \mid \pi \cdot f=f \text { for all } \pi \in S_{n}\right\}
$$

is the $\left(\begin{array}{c}n+|X|-1 \\ n\end{array}\right)$ dimensional complex Hilbert space of all symmetric functions on $X^{n}$ with inner product

$$
\langle f, g\rangle_{\mathcal{B}_{n}}=\int_{X^{n}} d \mathbf{x}_{1} \cdots d \mathbf{x}_{n} \overline{f\left(\mathbf{x}_{1}, \cdots, \mathbf{x}_{n}\right)} g\left(\mathbf{x}_{1}, \cdots, \mathbf{x}_{n}\right)
$$

In particular, $\mathcal{B}_{0}(X)=\mathbb{C}$ and $\mathcal{B}_{1}(X)=L^{2}(X)$.

(ii) The bosonic Fock space $\mathcal{B}(X)$ over $X$ is the orthogonal direct sum $\mathcal{B}(X)=\bigoplus_{n \geq 0} \mathcal{B}_{n}(X)$. It is an infinite dimensional complex Hilbert space. The inner product between $\mathbf{f}$ and $\mathbf{g}$ in $\mathcal{B}(X)$ is

$$
\langle\mathbf{f}, \mathbf{g}\rangle_{\mathcal{B}}=\sum_{n \geq 0}\left\langle f_{n}, g_{n}\right\rangle_{\mathcal{B}_{n}}
$$

\section{Definition II.2}

(i) Let $\mathrm{h}$ be a (single particle) operator on $L^{2}(X)$, with kernel $h(\mathbf{x}, \mathbf{y})$. Assume that $\mathrm{h}(\mathbf{y}, \mathbf{x})=$ $\mathrm{h}(\mathbf{x}, \mathbf{y})^{*}$ so that $\mathrm{h}$ is self-adjoint. The corresponding independent particle operator on $\mathcal{B}_{n}(X)$ is

$$
\mathrm{H}_{0}(\mathrm{~h}, n, X)=\sum_{i=1}^{n} \mathrm{~h}^{(i)}
$$

The superscript on $\mathrm{h}^{(i)}, i=1, \cdots, n$, indicates that the single particle operator $\mathrm{h}$ acts on the variable $\mathbf{x}_{i}$ appearing in a function $g\left(\mathbf{x}_{1}, \cdots, \mathbf{x}_{n}\right)$. That is,

$$
\left(\mathrm{h}^{(i)} g\right)\left(\mathbf{x}_{1}, \cdots, \mathbf{x}_{n}\right)=\int_{X} d \mathbf{x}_{i}^{\prime} \mathrm{h}\left(\mathbf{x}_{i}, \mathbf{x}_{i}^{\prime}\right) g\left(\mathbf{x}_{1}, \cdots, \mathbf{x}_{i}^{\prime}, \cdots, \mathbf{x}_{n}\right)
$$


By convention, $\mathrm{H}_{0}(\mathrm{~h}, 0, X)=0$. The kernel of $\mathrm{H}_{0}(\mathrm{~h}, n, X)$ is $\mathrm{H}_{0}(\mathrm{~h}, n, X)\left(\mathbf{x}_{1}, \cdots \mathbf{x}_{n}, \mathbf{y}_{1}, \cdots \mathbf{y}_{n}\right)=\sum_{i=1}^{n} \delta_{\mathbf{x}_{1}}\left(\mathbf{y}_{1}\right) \cdots \delta_{\mathbf{x}_{i-1}}\left(\mathbf{y}_{i-1}\right) \mathrm{h}\left(\mathbf{x}_{i}, \mathbf{y}_{i}\right) \delta_{\mathbf{x}_{i+1}}\left(\mathbf{y}_{i+1}\right) \cdots \delta_{\mathbf{x}_{n}}\left(\mathbf{y}_{n}\right)$ Here $\delta_{\mathbf{x}}(\mathbf{y})=\delta_{\mathbf{x}, \mathbf{y}}=\left\{\begin{array}{ll}1 & \text { if } \mathbf{x}=\mathbf{y} \\ 0 & \text { if } \mathbf{x} \neq \mathbf{y}\end{array}\right\}$ is the delta function on $X$ concentrated at the point $\mathbf{x}$. The second quantization of $\mathrm{h}$ is the direct sum

$$
\mathrm{H}_{0}(\mathrm{~h}, X)=\bigoplus_{n \geq 0} \mathrm{H}_{0}(\mathrm{~h}, n, X)
$$

acting on the domain $\left\{\mathbf{f} \in \mathcal{B}(X) \mid \sum_{n \geq 0}\left\|\mathrm{H}_{0}(\mathrm{~h}, n, X) \mathrm{f}_{n}\right\|_{\mathcal{B}_{n}}^{2}<\infty\right\}$.

(ii) Let $v(\mathbf{x}, \mathbf{y})$ be a real valued (two body) potential on $X^{2}$ satisfying $v(\mathbf{x}, \mathbf{y})=v(\mathbf{y}, \mathbf{x})$ for all $\mathbf{x}, \mathbf{y} \in X$. Multiplication by $v$ determines a (two particle) operator on $\mathcal{B}_{2}(X)$. Its kernel is $v\left(\mathbf{x}_{1}, \mathbf{x}_{2}\right) \delta_{\mathbf{x}_{1}}\left(\mathbf{y}_{1}\right) \delta_{\mathbf{x}_{2}}\left(\mathbf{y}_{2}\right)$. The corresponding 2 particle interaction operator on $\mathcal{B}_{n}(X)$ is

$$
\mathrm{V}(v, n, X)=\sum_{i_{1}<i_{2}} v^{\left(i_{1}, i_{2}\right)}
$$

Here, for each pair of indices $1 \leq i_{1}<i_{2} \leq n$, the superscript on $v^{\left(i_{1}, i_{2}\right)}$ indicates that the operator $v$ acts on the variables $\mathbf{x}_{i_{1}}, \mathbf{x}_{i_{2}}$ appearing in a function $g\left(\mathbf{x}_{1}, \cdots, \mathbf{x}_{n}\right)$. That is,

$$
\left(v^{\left(i_{1}, i_{2}\right)} g\right)\left(\mathbf{x}_{1}, \cdots, \mathbf{x}_{n}\right)=v\left(\mathbf{x}_{i_{1}}, \mathbf{x}_{i_{2}}\right) g\left(\mathbf{x}_{1}, \cdots, \mathbf{x}_{i_{1}}, \cdots, \mathbf{x}_{i_{2}}, \cdots, \mathbf{x}_{n}\right)
$$

By convention, $\mathrm{V}(v, n, X)=0$ for $n=0,1$.

The second quantization of the two particle interaction $v$ is the direct sum

$$
\mathrm{V}(v, X)=\bigoplus_{n \geq 0} \mathrm{~V}(v, n, X)
$$

acting on the domain $\left\{\mathbf{f} \in \mathcal{B}(X) \mid \sum_{n \geq 0}\left\|\mathrm{~V}(v, n, X) \mathrm{f}_{n}\right\|_{\mathcal{B}_{n}}^{2}<\infty\right\}$.

Example II.3 Let $\mathbb{1}_{X}$ be the identity operator on $L^{2}(X)$. Then,

$$
\mathrm{H}_{0}\left(\mathbb{1}_{X}, n, X\right)=n \mathbb{1}_{X^{n}}
$$

where, $\mathbb{1}_{X^{n}}$ is the identity operator on $L^{2}\left(X^{n}\right)$. By definition, the number operator $N$ is $\mathrm{H}_{0}\left(1_{X}, X\right)$.

Proposition II.4 For any single particle operator $\mathrm{h}$ on $X$, any two particle potential $v$ on $X$ and any $\mu \in \mathbb{R}$, the operator $\mathrm{H}_{0}(\mathrm{~h}, X)+\mathrm{V}(v, X)-\mu N$ is essentially self adjoint on $\left\{\left(f_{n}\right)_{n \geq 0} \mid f_{n} \in \mathcal{B}_{n}(X)\right.$, for all $n \geq 0, f_{n}=0$ for all but finitely many $\left.n\right\}$. It commutes with the number operator $N$.

Proof: The proof is routine. 


\section{Creation and Annihilation Operators}

Let $u \in \mathcal{B}_{m}(X)$ and let $v \in \mathcal{B}_{n}(X)$ and write $u \otimes v\left(\mathbf{x}_{1}, \cdots, \mathbf{x}_{m+n}\right)$ for the tensor product

$$
u\left(\mathbf{x}_{1}, \cdots, \mathbf{x}_{m}\right) v\left(\mathbf{x}_{m+1}, \cdots, \mathbf{x}_{m+n}\right)
$$

The symmetric tensor product $u \otimes_{s} v$ of $u$ and $v$ is defined by

$$
u \otimes_{s} v=\frac{1}{\sqrt{m !(m+n) ! n !}} \sum_{\pi \in S_{m+n}} \pi \cdot(u \otimes v)
$$

and belongs to $\mathcal{B}_{m+n}(X)$. The symmetric tensor product extends by linearity to a commutative, associative and distributive multiplication on $\mathcal{B}(X)$. If $u \in \mathcal{B}_{1}(X)$ and $v \in \mathcal{B}_{n-1}(X)$, then

$$
u \otimes_{s} v\left(\mathbf{x}_{1}, \cdots, \mathbf{x}_{n}\right)=\frac{1}{\sqrt{n}} \sum_{i=1}^{n} u\left(\mathbf{x}_{i}\right) v\left(\mathbf{x}_{1}, \cdots, \widehat{\mathbf{x}}_{i}, \cdots, \mathbf{x}_{n}\right)
$$

Successively multiplying $u_{1}, \cdots, u_{n} \in \mathcal{B}_{1}(X)$, one obtains by induction the element

$$
u_{1} \otimes_{s} \cdots \otimes_{s} u_{n}\left(\mathbf{x}_{1}, \cdots, \mathbf{x}_{n}\right)=\frac{1}{\sqrt{n !}} \sum_{\pi \in S_{n}} u_{1}\left(\mathbf{x}_{\pi(1)}\right) \cdots u_{n}\left(\mathbf{x}_{\pi(n)}\right)=\frac{1}{\sqrt{n !}} \operatorname{perm}\left(u_{i}\left(\mathbf{x}_{j}\right)\right)
$$

of $\mathcal{B}_{n}(X)$. Here perm $\left(u_{i}\left(\mathbf{x}_{j}\right)\right)$ denotes the permanent of the matrix $\left[u_{i}\left(\mathbf{x}_{j}\right)\right]_{1 \leq i, j \leq n}$. By direct calculation,

$$
\left\langle u_{1} \otimes_{s} \cdots \otimes_{s} u_{n}, v_{1} \otimes_{s} \cdots \otimes_{s} v_{n}\right\rangle_{\mathcal{B}_{n}}=\operatorname{perm}\left(\left\langle u_{i}, v_{j}\right\rangle_{\mathcal{B}_{1}}\right)
$$

for all $u_{1}, \cdots, u_{n}$ and $v_{1}, \cdots, v_{n}$ in $\mathcal{B}_{1}(X)$.

Remark II.5 It is common to define the symmetric tensor product with the alternative combinatorial factors $\frac{1}{(m+n) !}$ or $\frac{1}{m ! n !}$ rather than $\frac{1}{\sqrt{m !(m+n) ! n !}}$. We make the present choice because the annihilation operators become derivations on the algebra $\mathcal{B}(X)$. See, Lemma II.11.

Proposition II.6 Suppose $u_{i}, i=1, \cdots,|X|$, is an orthonormal basis for $\mathcal{B}_{1}(X)$. Then, the family of functions

$$
\left\{\frac{1}{\sqrt{\alpha !}} u^{\otimes_{s}^{\alpha}}\left|\alpha \in(\mathbb{N} \cup\{0\})^{|X|},\right| \alpha \mid=n\right\}
$$

where

$$
u^{\otimes_{s}^{\alpha}}\left(\mathbf{x}_{1}, \cdots, \mathbf{x}_{n}\right)=u_{1}^{\otimes_{s}^{\alpha_{1}}} \otimes_{s} \cdots \otimes_{s} u_{|X|}^{\otimes_{s}^{\alpha_{|X|}}}\left(\mathbf{x}_{1}, \cdots, \mathbf{x}_{n}\right)
$$

is an orthonormal basis for $\mathcal{B}_{n}(X)$.

If $Y$ is a subset of $X$, then $\mathcal{B}(Y)$ can be identified with the subalgebra

$$
\left\{\mathbf{f}=\left(f_{0}, f_{1}, \cdots\right) \in \mathcal{B}(X) \mid f_{n}\left(\mathbf{x}_{1}, \cdots, \mathbf{x}_{n}\right) \text { is supported in } Y^{n} \text { for each } n \geq 0\right\}
$$

of $\mathcal{B}(X)$. Furthermore, 
Lemma II.7 Let $Y$ and $Z$ be disjoint subsets of $X$. For all $u, u^{\prime} \in \mathcal{B}(Y)$ and $v, v^{\prime} \in \mathcal{B}(Z)$

$$
\left\langle u \otimes_{s} v, u^{\prime} \otimes_{s} v^{\prime}\right\rangle_{\mathcal{B}(X)}=\left\langle u, u^{\prime}\right\rangle_{\mathcal{B}(Y)}\left\langle v, v^{\prime}\right\rangle_{\mathcal{B}(Z)}
$$

Remark II.8 For each $\mathbf{x} \in X$, the bosonic Fock space $\mathcal{B}(\{\mathbf{x}\})$ is canonically isomorphic to a subalgebra of $\mathcal{B}(X)$. The abstract tensor product of all these subalgebras is isomorphic to $\mathcal{B}(X)$. By the last lemma, the global inner product completely factors.

Definition II.9 For each $\mathbf{x} \in X$ the annihilation operator $\psi(\mathbf{x})$ acts on $\mathcal{B}(X)$ by

$$
(\psi(\mathbf{x}) u)\left(\mathbf{x}_{1}, \cdots, \mathbf{x}_{n-1}\right)=\sqrt{n} u\left(\mathbf{x}, \mathbf{x}_{1}, \cdots, \mathbf{x}_{n-1}\right)
$$

for all $u \in \mathcal{B}_{n}(X), n \geq 0$. The adjoint $\psi^{\dagger}(\mathbf{x})$ of $\psi(\mathbf{x})$ is the creation operator on $\mathcal{B}(X)$ that acts by

$$
\begin{aligned}
\left(\psi^{\dagger}(\mathbf{x}) u\right)\left(\mathbf{x}_{1}, \cdots, \mathbf{x}_{n+1}\right) & =\left(\delta_{\mathbf{x}} \otimes_{s} u\right)\left(\mathbf{x}_{1}, \cdots, \mathbf{x}_{n+1}\right) \\
& =\frac{1}{\sqrt{n+1}} \sum_{i=1}^{n+1} \delta_{\mathbf{x}}\left(\mathbf{x}_{i}\right) u\left(\mathbf{x}_{1}, \cdots, \widehat{\mathbf{x}}_{i}, \cdots, \mathbf{x}_{n+1}\right)
\end{aligned}
$$

for all $u \in \mathcal{B}_{n}(X), n \geq 0$. The utility of these operators is due to the commutation relations

$$
\begin{aligned}
{\left[\psi(\mathbf{x}), \psi^{\dagger}\left(\mathbf{x}^{\prime}\right)\right] } & =\delta_{\mathbf{x}}\left(\mathbf{x}^{\prime}\right) \\
{\left[\psi(\mathbf{x}), \psi\left(\mathbf{x}^{\prime}\right)\right] } & =0 \\
{\left[\psi^{\dagger}(\mathbf{x}), \psi^{\dagger}\left(\mathbf{x}^{\prime}\right)\right] } & =0
\end{aligned}
$$

for all $\mathbf{x}, \mathbf{x}^{\prime} \in X$.

Remark II.10 It is easy to see that

$$
\begin{aligned}
\left(\psi^{\dagger}(\mathbf{x}) \psi(\mathbf{x}) u\right)\left(\mathbf{x}_{1}, \cdots, \mathbf{x}_{n}\right) & =\sum_{i=1}^{n} \delta_{\mathbf{x}}\left(\mathbf{x}_{i}\right) u\left(\mathbf{x}_{1}, \cdots, \mathbf{x}, \cdots, \mathbf{x}_{n}\right) \\
& =\left(\sum_{i=1}^{n} \delta_{\mathbf{x}}\left(\mathbf{x}_{i}\right)\right) u\left(\mathbf{x}_{1}, \cdots, \mathbf{x}_{i}, \cdots, \mathbf{x}_{n}\right)
\end{aligned}
$$

In other words, the restriction of the product $\psi^{\dagger}(\mathbf{x}) \psi(\mathbf{x})$ to $\mathcal{B}_{n}(X)$ is multiplication by the function $\sum_{i=1}^{n} \delta_{\mathbf{x}}\left(\mathbf{x}_{i}\right)$. By definition the density operator $n(\mathbf{x})$ at $\mathbf{x} \in X$ acts by

$$
n(\mathbf{x})=\psi^{\dagger}(\mathbf{x}) \psi(\mathbf{x})
$$

Lemma II.11 For all $\mathbf{x} \in X, u \in \mathcal{B}_{m}(X)$ and $v \in \mathcal{B}_{n}(X)$,

$$
\psi(\mathbf{x})\left(u \otimes_{s} v\right)=(\psi(\mathbf{x}) u) \otimes_{s} v+u \otimes_{s}(\psi(\mathbf{x}) v)
$$

Proof: The proof is by induction on $m$. 
Definition II.12 Fix a natural number $n \geq 0$. For each $Y$ in the quotient space $X^{n} / S_{n}$ set

$$
\delta_{Y}=c_{Y} \delta_{\mathbf{y}_{1}} \otimes_{s} \cdots \otimes_{s} \delta_{\mathbf{y}_{n}}
$$

where $\left(\mathbf{y}_{1}, \cdots, \mathbf{y}_{n}\right)$ in $X^{n}$ is a representative for $Y$. The constant

$$
c_{Y}=\frac{1}{\prod_{\mathbf{x} \in X} \sqrt{\mu_{Y}(\mathbf{x}) !}} \quad \text { where } \quad \mu_{Y}(\mathbf{x})=\sum_{i=1}^{n} \delta_{\mathbf{x}, \mathbf{y}_{i}}
$$

is the multiplicity of $\mathbf{x}$ in $Y$. By construction, $\delta_{\mathbf{y}}, \mathbf{y} \in X$, is an orthonormal basis for $L^{2}(X)$. By Proposition II.6, the family $\delta_{Y}, Y \in X^{n} / S_{n}$, is an orthonormal basis for $\mathcal{B}_{n}$.

Lemma II.13 Fix the point $\mathbf{x} \in X$ and the set $Y \in X^{n} / S_{n}$. Then,

$$
\begin{aligned}
\psi(\mathbf{x}) \delta_{Y} & = \begin{cases}c_{Y}^{-1} c_{Y \backslash\{\mathbf{x}\}} \delta_{Y \backslash\{\mathbf{x}\}} & \text { if } \mathbf{x} \in Y \\
0 & \text { if } \mathbf{x} \notin Y\end{cases} \\
\psi^{\dagger}(\mathbf{x}) \delta_{Y} & =c_{Y} c_{Y \sqcup\{\mathbf{x}\}}^{-1} \delta_{Y \sqcup\{\mathbf{x}\}} \\
n(\mathbf{x}) \delta_{Y} & =\mu_{Y}(\mathbf{x}) \delta_{Y}
\end{aligned}
$$

Here, the 'disjoint union' $Y \sqcup\{\mathbf{x}\}$ is the element of $X^{n+1} / S_{n+1}$ with

$$
\mu_{Y \sqcup\{\mathbf{x}\}}(\mathbf{y})= \begin{cases}\mu_{Y}(\mathbf{y}) & \text { if } \mathbf{y} \neq \mathbf{x} \\ \mu_{Y}(\mathbf{y})+1 & \text { if } \mathbf{y}=\mathbf{x}\end{cases}
$$

Proposition II.14 For any single particle operator $\mathrm{h}$ and any 2 particle potential $v$,

$$
\begin{aligned}
\mathrm{H}_{0}(\mathrm{~h}, X) & =\int d \mathbf{x} d \mathbf{y} \psi^{\dagger}(\mathbf{x}) \mathrm{h}(\mathbf{x}, \mathbf{y}) \psi(\mathbf{y}) \\
\mathrm{V}(v, X) & =\frac{1}{2} \int d \mathbf{x}_{1} d \mathbf{x}_{2} \psi^{\dagger}\left(\mathbf{x}_{1}\right) \psi^{\dagger}\left(\mathbf{x}_{2}\right) v\left(\mathbf{x}_{1}, \mathbf{x}_{2}\right) \psi\left(\mathbf{x}_{1}\right) \psi\left(\mathbf{x}_{2}\right) \\
& =\frac{1}{2} \int d \mathbf{x}_{1} d \mathbf{x}_{2} \psi^{\dagger}\left(\mathbf{x}_{1}\right) \psi\left(\mathbf{x}_{1}\right) v\left(\mathbf{x}_{1}, \mathbf{x}_{2}\right) \psi^{\dagger}\left(\mathbf{x}_{2}\right) \psi\left(\mathbf{x}_{2}\right)-\frac{1}{2} \int d \mathbf{x} \psi^{\dagger}(\mathbf{x}) v(\mathbf{x}, \mathbf{x}) \psi(\mathbf{x})
\end{aligned}
$$

Remark II.15 We have

$$
N=\mathrm{H}_{0}(\mathbb{1}, X)=\int d \mathbf{x} \psi^{\dagger}(\mathbf{x}) \psi(\mathbf{x})=\int d \mathbf{x} n(\mathbf{x})
$$

and

$$
\mathrm{V}(v, X)=\frac{1}{2} \int d \mathbf{x}_{1} d \mathbf{x}_{2} n\left(\mathbf{x}_{1}\right) v\left(\mathbf{x}_{1}, \mathbf{x}_{2}\right) n\left(\mathbf{x}_{2}\right)-\frac{1}{2} \int d \mathbf{x} v(\mathbf{x}, \mathbf{x}) n(\mathbf{x})
$$

To ensure that the Hamiltonian $H_{0}(\mathrm{~h}, X)+V(v, X)$ is stable, we shall assume that the interaction potential $v$ is repulsive in the sense of the following definition. 
Definition II.16 Define, for any real, symmetric, 2 particle potential $v(\mathbf{x}, \mathbf{y})$,

$$
\lambda_{0}(v)=\inf \left\{\int d \mathbf{x} d \mathbf{y} \rho(\mathbf{x}) v(\mathbf{x}, \mathbf{y}) \rho(\mathbf{y}) \mid \int d \mathbf{x} \rho(\mathbf{x})^{2}=1, \rho(\mathbf{x}) \geq 0 \text { for all } \mathbf{x} \in X\right\}
$$

We call the potential $v$ repulsive if $\lambda_{0}(v)>0$.

If $v(\mathbf{x}, \mathbf{y})$ is the kernel of a strictly positive definite operator acting on $L^{2}(X)$, then $v$ is repulsive with $\lambda_{0}(v)$ at least as large as the smallest eigenvalue of the operator. If $v(\mathbf{x}, \mathbf{y}) \geq 0$ for all $\mathbf{x}, \mathbf{y} \in X$ and $v(\mathbf{x}, \mathbf{x})>0$ for all $\mathbf{x} \in X$, then $v$ is repulsive with $\lambda_{0}(v) \geq \min _{\mathbf{x} \in X} v(\mathbf{x}, \mathbf{x})$.

Proposition II.17 Let $\mathrm{h}$ be a single particle operator and $v\left(\mathbf{x}_{1}, \mathbf{x}_{2}\right)$ be a real, symmetric, pair potential. Assume that the self adjoint operator $v$ acting on $L^{2}(X)$ with kernel $v(\mathbf{x}, \mathbf{y})$ is strictly positive definite. Then

$$
\begin{aligned}
-\frac{v_{0}^{2}}{8 \lambda_{0}}|X| \leq \frac{1}{2}\left(\lambda_{0} \frac{N}{|X|}-v_{0}\right) N & \leq \mathrm{V}(v, X) \leq \frac{1}{2}\left(\Lambda N-\lambda_{0}\right) N \\
\lambda_{0}^{\prime} N & \leq \mathrm{H}_{0}(\mathrm{~h}, X) \leq \Lambda^{\prime} N
\end{aligned}
$$

on the domain $\mathcal{D}(V)=\mathcal{D}\left(N^{2}\right)$. Here, $\lambda_{0}=\lambda_{0}(v), \Lambda$ is the largest eigenvalues of the operator $v, \lambda_{0}^{\prime}$ and $\Lambda^{\prime}$ are the smallest and largest eigenvalues of the operator $\mathrm{h}$ and $v_{0}=\max _{\mathbf{x} \in X} v(\mathbf{x}, \mathbf{x})$. The leftmost bound on $\mathrm{V}$ is called the 'linear lower bound'.

Proof: We have

$$
\begin{aligned}
\left\langle\delta_{Y}, \int_{X^{2}} d \mathbf{x}_{1} d \mathbf{x}_{2} n\left(\mathbf{x}_{1}\right) v\left(\mathbf{x}_{1}, \mathbf{x}_{2}\right) n\left(\mathbf{x}_{2}\right) \delta_{Y}\right\rangle & =\int_{X^{2}} d \mathbf{x}_{1} d \mathbf{x}_{2} \mu_{Y}\left(\mathbf{x}_{1}\right) v\left(\mathbf{x}_{1}, \mathbf{x}_{2}\right) \mu_{Y}\left(\mathbf{x}_{2}\right) \\
& \geq \lambda_{0} \int_{X} d \mathbf{x} \mu_{Y}^{2}(\mathbf{x}) \\
& \geq \frac{\lambda_{0}}{|X|}\left(\int_{X} d \mathbf{x} \mu_{Y}(\mathbf{x})\right)^{2} \\
& =\frac{\lambda_{0}}{|X|} n^{2}
\end{aligned}
$$

where Schwarz's inequality was used in the third line. Similarly,

$$
\left\langle\delta_{Y}, \int_{X^{2}} d \mathbf{x}_{1} d \mathbf{x}_{2} n\left(\mathbf{x}_{1}\right) v\left(\mathbf{x}_{1}, \mathbf{x}_{2}\right) n\left(\mathbf{x}_{2}\right) \delta_{Y}\right\rangle \leq \Lambda n^{2}
$$


Let $u=\sum_{Y \in X^{n} / S_{n}} \varphi_{Y} \delta_{Y}$ be an arbitrary element of $\mathcal{B}_{n}(X)$. We have

$$
\begin{aligned}
\left\langle u, \int_{X^{2}} d \mathbf{x}_{1} d \mathbf{x}_{2}\right. & \left.n\left(\mathbf{x}_{1}\right) v\left(\mathbf{x}_{1}, \mathbf{x}_{2}\right) n\left(\mathbf{x}_{2}\right) u\right\rangle \\
& =\sum_{Y_{1}, Y_{2} \in X^{n} / S_{n}} \varphi_{Y_{1}}^{*} \varphi_{Y_{2}}\left\langle\delta_{Y_{1}}, \int_{X^{2}} d \mathbf{x}_{1} d \mathbf{x}_{2} n\left(\mathbf{x}_{1}\right) v\left(\mathbf{x}_{1}, \mathbf{x}_{2}\right) n\left(\mathbf{x}_{2}\right) \delta_{Y_{2}}\right\rangle \\
& =\sum_{Y \in X^{n} / S_{n}}\left|\varphi_{Y}\right|^{2} \int_{X^{2}} d \mathbf{x}_{1} d \mathbf{x}_{2} \mu_{Y}\left(\mathbf{x}_{1}\right) v\left(\mathbf{x}_{1}, \mathbf{x}_{2}\right) \mu_{Y}\left(\mathbf{x}_{2}\right) \\
& \geq \frac{\lambda_{0}}{|X|} n^{2} \sum_{Y \in X^{n} / S_{n}}\left|\varphi_{Y}\right|^{2} \\
& =\frac{\lambda_{0}}{|X|} n^{2}\|u\|_{\mathcal{B}_{n}}^{2}
\end{aligned}
$$

In the same way,

$$
\left\langle u, \int_{X^{2}} d \mathbf{x}_{1} d \mathbf{x}_{2} n\left(\mathbf{x}_{1}\right) v\left(\mathbf{x}_{1}, \mathbf{x}_{2}\right) n\left(\mathbf{x}_{2}\right) u\right\rangle \leq \Lambda n^{2}\|u\|_{\mathcal{B}_{n}}^{2}
$$

Since $a \leq v(x, x) \leq b$, a similar, but simpler, argument gives

$$
\lambda_{0} n\|u\|_{\mathcal{B}_{n}}^{2} \leq\left\langle u, \int_{X} d \mathbf{x} v(\mathbf{x}, \mathbf{x}) n(\mathbf{x}) u\right\rangle \leq v_{0} n\|u\|_{\mathcal{B}_{n}}^{2}
$$

The bound on $\mathrm{H}_{0}(\mathrm{~h}, X)$ follows directly from Definition II.2.i.

\section{Coherent States}

We now review the formalism of coherent states [NO, $§ 1.5]$.

\section{Definition II.18}

(i) The family $\left|z \delta_{\mathbf{x}}\right\rangle, z \in \mathbb{C}$, of coherent states concentrated at $\mathbf{x} \in X$ is given by

$$
\left|z \delta_{\mathbf{x}}\right\rangle=\sum_{n \geq 0} \frac{1}{n !} z^{n} \psi^{\dagger}(\mathbf{x})^{n} \mathbf{1}=\sum_{n \geq 0} \frac{1}{n !} z^{n} \delta_{\mathbf{x}}^{\otimes_{s}^{n}}
$$

where 1 is the 'vacuum' $1 \in \mathbb{C}=\mathcal{B}_{0}(X)$. That $\left|z \delta_{\mathbf{x}}\right\rangle \in \mathcal{B}(X)$ is a consequence of Proposition II.22, below.

(ii) If $\phi(\mathbf{y}) \in L^{2}(X)$, the coherent state $|\phi\rangle \in \mathcal{B}(X)$ is

$$
|\phi\rangle=\bigotimes_{\mathbf{y} \in X}\left|\phi(\mathbf{y}) \delta_{\mathbf{y}}\right\rangle=e^{\int d \mathbf{y} \phi(\mathbf{y}) \psi^{\dagger}(\mathbf{y})} \mathbf{1}
$$


Lemma II.19 For all $\phi$ in $L^{2}(X)$,

$$
|\phi\rangle=\sum_{n \geq 0} \sum_{Y \in X^{n} / S_{n}} \phi(Y) c_{Y} \delta_{Y}
$$

Here, $\phi(Y)=\prod_{\mathbf{y} \in X} \phi^{\mu_{Y}(\mathbf{y})}(\mathbf{y})$ for each $Y$ in the quotient space $X^{n} / S_{n}$.

Proof:

$$
\begin{aligned}
|\phi\rangle & =\bigotimes_{\mathbf{y} \in X} \sum_{n \geq 0} \frac{1}{n !} \phi^{n}(\mathbf{y}) \delta_{\mathbf{y}}^{\otimes_{s}^{n}}=\sum_{\substack{n \mathbf{y} \geq 0 \\
\mathbf{y} \in X}}\left(\prod_{\mathbf{y} \in X} \frac{1}{n_{\mathbf{y}} !} \phi^{n_{\mathbf{y}}}(\mathbf{y})\right) \bigotimes_{\mathbf{y} \in X} \delta_{\mathbf{y}}^{\otimes_{s}^{n_{\mathbf{y}}}} \\
& =\sum_{n \geq 0} \sum_{\substack{n_{\mathbf{y} \geq 0} \geq 0 \\
\mathbf{y} \in X}} \chi\left(n=\sum_{y \in X} n_{\mathbf{y}}\right)\left(\prod_{\mathbf{y} \in X} \frac{1}{n_{\mathbf{y}} !} \phi^{n_{\mathbf{y}}}(\mathbf{y})\right) \bigotimes_{\mathbf{y} \in X} \delta_{\mathbf{y}}^{\bigotimes_{s}^{n_{\mathbf{y}}}} \\
& =\sum_{n \geq 0} \sum_{\substack{Y \in X^{n} / S_{n}\\
}}\left(\prod_{\mathbf{y} \in X} \phi^{\mu_{Y}(\mathbf{y})}(\mathbf{y})\right) c_{Y}^{2} \bigotimes_{\mathbf{y} \in X} \delta_{\mathbf{y}}^{\otimes_{s}^{\mu_{Y}(\mathbf{y})}} \\
& =\sum_{n \geq 0} \sum_{Y \in X^{n} / S_{n}} \phi(Y) c_{Y} \delta_{Y}
\end{aligned}
$$

Coherent states have been defined to give

Proposition II.20 For all $\mathrm{x} \in X$ and $\phi \in L^{2}(X)$,

$$
\begin{aligned}
\psi(\mathbf{x})|\phi\rangle & =\phi(\mathbf{x})|\phi\rangle \\
\psi^{\dagger}(\mathbf{x})|\phi\rangle & =\frac{\partial}{\partial \phi(\mathbf{x})}|\phi\rangle
\end{aligned}
$$

Convention II.21 For any $\phi$ in $L^{2}(X)$ and any state $\mathbf{f}$ in the Fock space $\mathcal{B}(X)$, abusing notation, the inner product between the coherent state $|\phi\rangle$ and $\mathbf{f}$ is written as $\langle\phi \mid \mathbf{f}\rangle$. That $i s,\langle\phi \mid \mathbf{f}\rangle=\langle\mid \phi\rangle, \mathbf{f}\rangle_{\mathcal{B}}$ Similarly, the inner product between the coherent states $|\phi\rangle$ and $\left|\phi^{\prime}\right\rangle$ is written $\left\langle\phi \mid \phi^{\prime}\right\rangle$.

Proposition II.22 For all $\alpha$ and $\gamma$ in $L^{2}(X)$, we have $\| P^{(m)}|\alpha\rangle \|_{\mathcal{B}_{m}}=\frac{\|\alpha\|^{m}}{\sqrt{m !}}$ and

$$
\langle\alpha \mid \gamma\rangle=e^{\int d \mathbf{y} \overline{\alpha(\mathbf{y})} \gamma(\mathbf{y})}
$$

where $P^{(m)}$ is the orthogonal projection from $\mathcal{B}(X)$ onto the $m$ particle subspace $\mathcal{B}_{m}(X)$. 
Lemma II.23 For any single particle operator $\mathrm{h}$ and any $\phi$ in $L^{2}(X)$,

$$
e^{-\tau \mathrm{H}_{0}(\mathrm{~h}, X)}|\phi\rangle=\left|e^{-\tau \mathrm{h}} \phi\right\rangle
$$

Proof: By Proposition II.14,

$$
\frac{d}{d \tau} e^{-\tau \mathrm{H}_{0}}|\phi\rangle=-\int d \mathbf{x}_{1} d \mathbf{x}_{2} \psi^{\dagger}\left(\mathbf{x}_{1}\right) \mathrm{h}\left(\mathbf{x}_{1}, \mathbf{x}_{2}\right) \psi\left(\mathbf{x}_{2}\right) e^{-\tau \mathrm{H}_{0}}|\phi\rangle
$$

and, by Definition II.18.ii,

$$
\begin{aligned}
\frac{d}{d \tau}\left|e^{-\tau \mathrm{h}} \phi\right\rangle & =\frac{\partial}{\partial \tau} e^{\int d \mathbf{y}\left(e^{-\tau \mathrm{h}} \phi\right)(\mathbf{y}) \psi^{\dagger}(\mathbf{y})} \mathbf{1} \\
& =\int d \mathbf{y}\left(-\mathrm{h} e^{-\tau \mathrm{h}} \phi\right)(\mathbf{y}) \psi^{\dagger}(\mathbf{y}) e^{\int d \mathbf{y}\left(e^{-\tau \mathrm{h}} \phi\right)(\mathbf{y}) \psi^{\dagger}(\mathbf{y})} \mathbf{1} \\
& =-\int d \mathbf{y} \psi^{\dagger}(\mathbf{y})\left(\mathrm{h} e^{-\tau \mathrm{h}} \phi\right)(\mathbf{y})\left|e^{-\tau \mathrm{h}} \phi\right\rangle \\
& =-\int d \mathbf{x}_{1} d \mathbf{x}_{2} \psi^{\dagger}\left(\mathbf{x}_{1}\right) \mathrm{h}\left(\mathbf{x}_{1}, \mathbf{x}_{2}\right) \psi\left(\mathbf{x}_{2}\right)\left|e^{-\tau \mathrm{h}} \phi\right\rangle
\end{aligned}
$$

As $\frac{d}{d \tau} e^{-\tau \mathrm{H}_{0}}|\phi\rangle$ and $\frac{d}{d \tau}\left|e^{-\tau \mathrm{h}} \phi\right\rangle$ satisfy the same first order ordinary differential equation and initial condition, they coincide.

Lemma II.24 Suppose both $\mathbf{f}$ and $N^{2|X|} \mathbf{f}$ belong to the Fock space $\mathcal{B}$. Then,

$$
|\langle\phi \mid \mathbf{f}\rangle| \leq\left(\prod_{\mathbf{y} \in X} \frac{1}{1+|\phi(\mathbf{y})|^{4}}\right) 2^{|X|} e^{\frac{1}{2}\|\phi\|^{2}}\left\|(N+4|X|)^{2|X|} \mathbf{f}\right\|_{\mathcal{B}}
$$

Proof: Fix $P$ in $X^{n} / S_{n}$. Then,

$$
\begin{aligned}
\phi(P)\langle\phi \mid \mathbf{f}\rangle & =\sum_{Y} C_{Y} \phi(P) \phi(Y)\left\langle\delta_{Y}, \mathbf{f}\right\rangle_{\mathcal{B}} \\
& =\sum_{Y} C_{P \sqcup Y} \phi(P \sqcup Y)\left\langle\frac{C_{Y}}{C_{P \sqcup Y}} \delta_{Y}, \mathbf{f}\right\rangle_{\mathcal{B}} \\
& =\sum_{Y} C_{P \sqcup Y} \phi(P \sqcup Y)\left\langle\left[\prod_{\mathbf{x} \in X} \frac{\mu_{P \sqcup Y}(\mathbf{x}) !}{\mu_{Y}(\mathbf{x}) !}\right]^{\frac{1}{2}} \delta_{Y}, \mathbf{f}\right\rangle_{\mathcal{B}} \\
& =\sum_{Y} C_{P \sqcup Y} \phi(P \sqcup Y)\left\langle\prod_{\mathbf{x} \in X} \prod_{k=1}^{\mu_{P}(\mathbf{x})}(n(\mathbf{x})+k)^{\frac{1}{2}} \delta_{Y}, \mathbf{f}\right\rangle_{\mathcal{B}} \\
& =\sum_{Y} C_{P \sqcup Y} \phi(P \sqcup Y)\left\langle\delta_{Y}, \prod_{\mathbf{x} \in X} \prod_{k=1}^{\mu_{P}(\mathbf{x})}(n(\mathbf{x})+k)^{\frac{1}{2}} \mathbf{f}\right\rangle_{\mathcal{B}}
\end{aligned}
$$


It follows from Schwarz's inequality and Parseval's identity that

$$
\begin{aligned}
|\phi(P)\langle\phi \mid \mathbf{f}\rangle| & \leq\left(\sum_{Y}\left|C_{P \sqcup Y} \phi(P \sqcup Y)\right|^{2}\right)^{\frac{1}{2}}\left\|\prod_{\mathbf{x} \in X} \prod_{k=1}^{\mu_{P}(\mathbf{x})}(n(\mathbf{x})+k)^{\frac{1}{2}} \mathbf{f}\right\|_{\mathcal{B}} \\
& \leq\left(\sum_{Y}\left|C_{Y} \phi(Y)\right|^{2}\right)^{\frac{1}{2}}\left\|\prod_{\mathbf{x} \in X} \prod_{k=1}^{\mu_{P}(\mathbf{x})}(n(\mathbf{x})+k)^{\frac{1}{2}} \mathbf{f}\right\|_{\mathcal{B}} \\
& \leq e^{\frac{1}{2}\|\phi\|^{2}}\left\|(N+n)^{\frac{n}{2}} \mathbf{f}\right\|_{\mathcal{B}}
\end{aligned}
$$

Adding,

$$
|\langle\phi \mid \mathbf{f}\rangle| \prod_{\mathbf{y} \in X}\left(1+|\phi(\mathbf{y})|^{4}\right) \leq 2^{|X|} e^{\frac{1}{2}\|\phi\|^{2}}\left\|(N+4|X|)^{2|X|} \mathbf{f}\right\|_{\mathcal{B}}
$$

\section{An Approximate Resolution of the Identity}

In the physics literature, the formal resolution of the identity

$$
\mathbb{1}=\int \prod_{\mathbf{x} \in X}\left[\frac{d \phi^{*}(\mathbf{x}) d \phi(\mathbf{x})}{2 \pi \imath}\right] e^{-\int d \mathbf{y}|\phi(\mathbf{y})|^{2}}|\phi\rangle\langle\phi|
$$

is often used. See, for example [NO, (1.123)]. However, for each $\phi \in L^{2}(T)$, the operator norm of $e^{-\int d \mathbf{y}|\phi(\mathbf{y})|^{2}}|\phi\rangle\langle\phi|$ is exactly one. So the nature of the convergence of the integral in the above formal resolution of the identity is not clear. We now investigate the convergence more carefully.

Definition II.25 For each $r>0$, the measure $d \mu_{r}\left(\phi^{*}, \phi\right)$ on $L^{2}(X)$ is given by

$$
d \mu_{r}\left(\phi^{*}, \phi\right)=\prod_{\mathbf{x} \in X}\left[\frac{d \phi^{*}(\mathbf{x}) d \phi(\mathbf{x})}{2 \pi \imath} \chi_{r}(|\phi(\mathbf{x})|)\right]
$$

where $\chi_{r}$ is the characteristic function of the interval $[0, r]$. The measure $d \mu\left(\phi^{*}, \phi\right)$ on $L^{2}(X)$ is given by

$$
d \mu\left(\phi^{*}, \phi\right)=\prod_{\mathbf{x} \in X}\left[\frac{d \phi^{*}(\mathbf{x}) d \phi(\mathbf{x})}{2 \pi \imath}\right]
$$

Theorem II.26 For each $r>0$, let $\mathrm{I}_{r}$ be the operator that acts on $\mathbf{f}$ in $\mathcal{B}(X)$ by

$$
\mathrm{I}_{r} \mathbf{f}=\int d \mu_{r}\left(\phi^{*}, \phi\right) e^{-\|\phi\|^{2}}|\phi\rangle\langle\phi \mid \mathbf{f}\rangle
$$

(a) For all $n \geq 0$ and all $Y \in X^{n} / S_{n}$,

$$
\mathrm{I}_{r} \delta_{Y}=\lambda_{r}(Y) \delta_{Y}
$$


where

$$
\lambda_{r}(Y)=\prod_{\mathbf{x} \in X} \frac{\Gamma_{r}\left(\mu_{Y}(\mathbf{x})\right)}{\mu_{Y}(\mathbf{x}) !} \quad \text { with } \quad \Gamma_{r}(s)=\int_{0}^{r^{2}} d t e^{-t} t^{s}, \text { for all } s>-1
$$

In particular, $0 \leq \lambda_{r}(Y) \leq 1$.

(b) $\mathrm{I}_{r}$ commutes with $N$.

(c) The operator norm of $\mathrm{I}_{r}$ is bounded by one for all $r$ and $\mathrm{I}_{r}$ converges strongly to the identity operator as $r \rightarrow \infty$.

(d) For all $n$ and $r$, the operator norm

$$
\left\|\left(1-\mathrm{I}_{r}\right) \mathrm{P}_{n}\right\| \leq|X| 2^{n+1} e^{-r^{2} / 2}
$$

Here, $\mathrm{P}_{n}$ is the orthogonal projection from $\mathcal{B}(X)$ onto the direct sum $\underset{0 \leq m \leq n}{\bigoplus} \mathcal{B}_{m}(X)$.

(e) Suppose that $N^{2|X|} \mathbf{f}$ belongs to the Fock space $\mathcal{B}$. Then,

$$
\mathbf{f}=\int d \mu\left(\phi^{*}, \phi\right) e^{-\|\phi\|^{2}}|\phi\rangle\langle\phi \mid \mathbf{f}\rangle
$$

with the integral converging absolutely.

Proof: (a,b) To verify (a), let $Y \in X^{n} / S_{n}$ and observe that

$$
\begin{aligned}
\mathrm{I}_{r} \delta_{Y} & =\int d \mu_{r}\left(\phi^{*}, \phi\right) e^{-\int d \mathbf{y}|\phi(\mathbf{y})|^{2}}|\phi\rangle\left\langle\phi \mid \delta_{Y}\right\rangle \\
& =\sum_{n \geq 0} \sum_{Y^{\prime} \in X^{n} / S_{n}} \delta_{Y^{\prime}} c_{Y^{\prime}} c_{Y} \int d \mu_{r}\left(\phi^{*}, \phi\right) e^{-\int d \mathbf{y}|\phi(\mathbf{y})|^{2}} \phi\left(Y^{\prime}\right) \phi^{*}(Y)
\end{aligned}
$$

We have

$$
\begin{aligned}
& \int d \mu_{r}\left(\phi^{*}, \phi\right) e^{-\int d \mathbf{y}|\phi(\mathbf{y})|^{2}} \phi^{*}(Y) \phi\left(Y^{\prime}\right) \\
& =\prod_{\mathbf{x} \in X}\left[\int \frac{d \phi^{*}(\mathbf{x}) d \phi(\mathbf{x})}{2 \pi \imath} \chi_{r}(|\phi(\mathbf{x})|) e^{-|\phi(\mathbf{x})|^{2}} \phi^{*}(\mathbf{x})^{\mu_{Y}(\mathbf{x})} \phi(\mathbf{x})^{\mu_{Y^{\prime}}(\mathbf{x})}\right] \\
& =\prod_{\mathbf{x} \in X}\left[\int_{0}^{r} d \rho \int_{0}^{2 \pi} \frac{d \theta}{\pi} \rho e^{-\rho^{2}} \rho^{\mu_{Y}(\mathbf{x})+\mu_{Y^{\prime}}}(\mathbf{x}) e^{\imath \theta\left(\mu_{Y^{\prime}}(\mathbf{x})-\mu_{Y}(\mathbf{x})\right)}\right] \\
& =\prod_{\mathbf{x} \in X}\left[\delta_{\mu_{Y}(\mathbf{x}), \mu_{Y^{\prime}}(\mathbf{x})} \int_{0}^{r} d \rho 2 \rho e^{-\rho^{2}} \rho^{2 \mu_{Y}(\mathbf{x})}\right] \\
& =\delta_{Y, Y^{\prime}} \prod_{\mathbf{x} \in X} \int_{0}^{r^{2}} d t e^{-t} t^{\mu_{Y}(\mathbf{x})} \\
& =\delta_{Y, Y^{\prime}} \prod_{\mathbf{x} \in X} \Gamma_{r}\left(\mu_{Y}(\mathbf{x})\right)
\end{aligned}
$$


Consequently,

$$
\mathrm{I}_{r} \delta_{Y}=\delta_{Y} c_{Y}^{2} \prod_{\mathbf{x} \in X} \Gamma_{r}\left(\mu_{Y}(\mathbf{x})\right)=\delta_{Y} \prod_{\mathbf{x} \in X} \frac{\Gamma_{r}\left(\mu_{Y}(\mathbf{x})\right)}{\mu_{Y}(\mathbf{x}) !}
$$

Thus $I_{r}$ is a diagonal operator in the orthonormal basis $\left\{\delta_{Y}\right\}$. Each diagonal entry

$$
\lambda_{r}(Y)=\prod_{\mathbf{x} \in X}\left[\frac{1}{\mu_{Y}(\mathbf{x}) !} \int_{0}^{r^{2}} d t e^{-t} t^{\mu_{Y}(\mathbf{x})}\right]
$$

is between 0 and 1 . Since $\left\{\delta_{Y}\right\}$ is a basis of eigenvectors for both $I_{r}$ and $N$, they commute, which proves parts (a) and (b).

(c) Each

$$
\lambda_{r}(Y)=\prod_{\mathbf{x} \in X}\left[\frac{1}{\mu_{Y}(\mathbf{x}) !} \int_{0}^{r^{2}} d t e^{-t} t^{\mu_{Y}(\mathbf{x})}\right]
$$

approaches 1 in the limit $r \rightarrow \infty$. By the Lebesgue dominated convergence theorem, if $f=\sum_{n} \sum_{Y \in X^{n} / S_{n}} f_{Y} \delta_{Y}$ is any vector in $\mathcal{B}(X)$, then

$$
\lim _{r \rightarrow \infty}\left\|f-\mathrm{I}_{r} f\right\|^{2}=\lim _{r \rightarrow \infty} \sum_{n} \sum_{Y \in X^{n} / S_{n}}\left(1-\lambda_{r}(Y)\right)^{2}\left|f_{Y}\right|^{2}=0
$$

The operator norm of $\mathrm{I}_{r}$ is bounded by 1 for all $r>0$, because all of the eigenvalues of $\mathrm{I}_{r}$ are between 0 and 1 . This completes the proof of part (c).

(d) We bound

$$
\left\|\left(\mathbb{1}-\mathrm{I}_{r}\right) \mathrm{P}_{n}\right\|=\max _{|Y| \leq n}\left[1-\lambda_{r}(Y)\right]
$$

Fix any $Y$ with $|Y| \leq n$ and set, for each $\mathbf{x} \in X$,

$$
\beta_{\mathbf{x}}=\frac{1}{\mu_{Y}(\mathbf{x}) !} \int_{r^{2}}^{\infty} d t e^{-t} t^{\mu_{Y}(\mathbf{x})}
$$

By (II.2)

$$
1-\lambda_{r}(Y)=1-\prod_{\mathbf{x} \in X}\left(1-\beta_{\mathbf{x}}\right) \leq \sum_{\mathbf{x} \in X} \beta_{\mathbf{x}} \leq|X| \max _{\mathbf{x} \in X} \beta_{\mathbf{x}}
$$

The claim now follows from

$$
\begin{aligned}
\beta_{\mathbf{x}} & =\frac{2^{\mu_{Y}(\mathbf{x})}}{\mu_{Y}(\mathbf{x}) !} \int_{r^{2}}^{\infty} d t e^{-t}\left(\frac{t}{2}\right)^{\mu_{Y}(\mathbf{x})} \leq 2^{\mu_{Y}(\mathbf{x})} \int_{r^{2}}^{\infty} d t e^{-t / 2}=2^{\mu_{Y}(\mathbf{x})+1} e^{-r^{2} / 2} \\
& \leq 2^{n+1} e^{-r^{2} / 2}
\end{aligned}
$$

(e) By definition

$$
\mathrm{I}_{r} \mathbf{f}=\int d \mu\left(\phi^{*}, \phi\right) \prod_{\mathbf{x} \in X} \chi_{r}(|\phi(\mathbf{x})|) e^{-\|\phi\|^{2}}|\phi\rangle\langle\phi \mid \mathbf{f}\rangle
$$


By part (c) the left hand side converges to $\mathbf{f}$ as $r \rightarrow \infty$. By Lemma II.24, the norm of the integrand of the right hand side is bounded by

$$
\left(\prod_{\mathbf{y} \in X} \frac{1}{1+|\phi(\mathbf{y})|^{4}}\right) 2^{|X|}\left\|(N+4|X|)^{2|X|} \mathbf{f}\right\|_{\mathcal{B}}
$$

which is integrable with respect to $d \mu\left(\phi^{*}, \phi\right)$. Hence, as $r \rightarrow \infty$, the right hand side converges to $\int d \mu\left(\phi^{*}, \phi\right) e^{-\|\phi\|^{2}}|\phi\rangle\langle\phi \mid \mathbf{f}\rangle$.

\section{The Trace Formula}

Another commonly used formal property of coherent states is the trace formula (I.4). We now develop a rigorous, but limited, version of this formula that is adequate for our purposes.

Remark II.27 We use the approximate identity $\mathrm{I}_{r}$ to prove a "cutoff" trace for any bounded operator that computes with $N$. By (II.2), for each fixed $Y \in X^{n} / S_{n}, \lim _{r \rightarrow \infty} \lambda_{r}(Y)=1$. On the other hand, for each fixed $r$, there is a constant $C_{r, X}=r^{2}|X|$ such that

$$
\max _{Y \in X^{n} / S_{n}} \lambda_{r}(Y) \leq \frac{1}{n !} C_{r, X}^{n}
$$

Thus the operator norm of $\mathrm{I}_{r}$, restricted to $\mathcal{B}_{n}(X)$, is bounded by $\frac{1}{n !} C_{r, X}^{n}$. The dimension of $\mathcal{B}_{n}(X)$ is bounded by $|X|^{n}$. Therefore, for any bounded operator $B$ that commutes with $N$, $B \mathrm{I}_{r}$ is trace class and

$$
\operatorname{Tr} B \mathrm{I}_{r}=\lim _{n \rightarrow \infty} \operatorname{Tr} B \mathrm{I}_{r} \mathrm{P}_{n}
$$

\section{Proposition II.28}

(a) Let $B$ be a bounded operator on $\mathcal{B}(X)$ that commutes with $N$. Then, for all $r>0, B \mathrm{I}_{r}$ is trace class and

$$
\operatorname{Tr} B \mathrm{I}_{r}=\int d \mu_{r}\left(\phi^{*}, \phi\right) e^{-\int d \mathbf{y}|\phi(\mathbf{y})|^{2}}\langle\phi|B| \phi\rangle
$$

(b) Let $B$ be any operator on $\mathcal{B}(X)$ that commutes with $N$ and obeys

$$
\left\|P^{(n)} B\right\|_{\mathcal{B}_{n}} \leq \operatorname{const}(1+n)^{-2|X|}
$$

Then $B$ is trace class and

$$
\operatorname{Tr} B=\int d \mu\left(\phi^{*}, \phi\right) e^{-\int d \mathbf{y}|\phi(\mathbf{y})|^{2}}\langle\phi|B| \phi\rangle
$$


Proof: (a) We have

$$
\begin{aligned}
\operatorname{Tr} B \mathrm{I}_{r} \mathrm{P}_{n} & =\sum_{\operatorname{deg} Y \leq n}\left\langle\delta_{Y}\left|B \mathrm{I}_{r}\right| \delta_{Y}\right\rangle \\
& =\sum_{\operatorname{deg} Y \leq n} \int d \mu_{r}\left(\phi^{*}, \phi\right) e^{-\int d \mathbf{y}|\phi(\mathbf{y})|^{2}}\left\langle\delta_{Y}|B| \phi\right\rangle\left\langle\phi \mid \delta_{Y}\right\rangle \\
& =\int d \mu_{r}\left(\phi^{*}, \phi\right) e^{-\int d \mathbf{y}|\phi(\mathbf{y})|^{2}} \sum_{\operatorname{deg} Y \leq n}\left\langle\phi \mid \delta_{Y}\right\rangle\left\langle\delta_{Y}|B| \phi\right\rangle \\
& =\int d \mu_{r}\left(\phi^{*}, \phi\right) e^{-\int d \mathbf{y}|\phi(\mathbf{y})|^{2}}\left\langle\phi\left|\mathrm{P}_{n} B\right| \phi\right\rangle
\end{aligned}
$$

Since $B$ is a bounded operator, $e^{-\int d \mathbf{y}|\phi(\mathbf{y})|^{2}}\left|\left\langle\phi\left|\mathrm{P}_{n} B\right| \phi\right\rangle\right|$ is bounded uniformly in $n$ and $\phi$ and the dominated convergence theorem provides the limit of the right hand side as $n \rightarrow \infty$. By Remark II.27, the left hand side converges to $\operatorname{Tr} B \mathrm{I}_{r}$ as $n \rightarrow \infty$.

(b) As in part (a), but using part (e) of Theorem II.26,

$$
\operatorname{Tr} B \mathrm{P}_{n}=\int d \mu\left(\phi^{*}, \phi\right) e^{-\int d \mathbf{y}|\phi(\mathbf{y})|^{2}}\left\langle\phi\left|\mathrm{P}_{n} B\right| \phi\right\rangle
$$

By Lemma II.24,

$$
\begin{aligned}
\left|\left\langle\phi\left|\mathrm{P}_{n} B\right| \phi\right\rangle\right| & \leq\left(\prod_{\mathbf{y} \in X} \frac{1}{1+|\phi(\mathbf{y})|^{4}}\right) 2^{|X|} e^{\frac{1}{2}\|\phi\|^{2}} \|(N+4|X|)^{2|X|} \mathrm{P}_{n} B|\phi\rangle \|_{\mathcal{B}} \\
& \leq e^{\|\phi\|^{2}}\left(\prod_{\mathbf{y} \in X} \frac{1}{1+|\phi(\mathbf{y})|^{4}}\right) 2^{|X|}\left\|(N+4|X|)^{2|X|} B\right\|
\end{aligned}
$$

By the Lebesgue dominated convergence theorem

$$
\lim _{n \rightarrow \infty} \int d \mu\left(\phi^{*}, \phi\right) e^{-\int d \mathbf{y}|\phi(\mathbf{y})|^{2}}\left\langle\phi\left|\mathrm{P}_{n} B\right| \phi\right\rangle=\int d \mu\left(\phi^{*}, \phi\right) e^{-\int d \mathbf{y}|\phi(\mathbf{y})|^{2}}\langle\phi|B| \phi\rangle
$$

Since the dimension of $\mathcal{B}_{n}(X)$ is $\frac{(n+|X|-1) !}{n !(|X|-1) !} \leq \frac{(n+|X|-1)|X|-1}{(|X|-1) !}$ and the operator norm of the restriction of $B$ to $\mathcal{B}_{n}(X)$ is bounded by a constant times $(1+n)^{-2|X|}, B$ is trace class and

$$
\lim _{n \rightarrow \infty} \operatorname{Tr} B \mathrm{P}_{n}=\operatorname{Tr} B
$$




\section{An Integral Representation of the Partition Function}

Let $\mathrm{h}$ be a single particle operator on $X$ and $v\left(\mathbf{x}_{1}, \mathbf{x}_{2}\right)$ a real, symmetric, pair potential which is repulsive in the sense of Definition II.16. For the rest of this paper, except where otherwise stated, we write

$$
K=K(\mathrm{~h}, v, X, \mu)=\mathrm{H}_{0}(\mathrm{~h}, X)+\mathrm{V}(v, X)-\mu N
$$

Recall that $\mathrm{H}_{0}(\mathrm{~h}, X)$ and $\mathrm{V}(v, X)$ were defined in Definition II.2.

The first step in the formal derivation of the functional integral representation (I.1) is the application of the resolution of the identity (I.3) and the trace formula (I.4) to give the intermediate representation (I.5). Theorem III.1, below, is a rigorous version of (I.5).

Theorem III.1 Suppose that the sequence $\mathrm{R}(p)$ obeys

$$
\lim _{p \rightarrow \infty} p e^{-\frac{1}{2} \mathrm{R}(p)^{2}}=0
$$

Then,

$$
\operatorname{Tr} e^{-\beta K}=\lim _{p \rightarrow \infty} \int \prod_{\tau \in \mathcal{T}_{p}}\left[d \mu_{\mathrm{R}(p)}\left(\phi_{\tau}^{*}, \phi_{\tau}\right) e^{-\int d \mathbf{y}\left|\phi_{\tau}(\mathbf{y})\right|^{2}}\right] \prod_{\tau \in \mathcal{T}_{p}}\left\langle\phi_{\tau-\varepsilon}\left|e^{-\varepsilon K}\right| \phi_{\tau}\right\rangle
$$

where $\mathcal{T}_{p}=\{\tau=q \varepsilon \mid q=1, \cdots, p\}$ and we use the conventions that $\varepsilon=\frac{\beta}{p}$ and $\phi_{0}=$ $\phi_{p \varepsilon}=\phi_{\beta}$.

In Proposition III.2, below, we prove that the grand canonical partition function $\operatorname{Tr} e^{-\beta K}$ is well-defined. Then we prove Lemma III.4, which provides a rigorous multiple insertion of the approximate resolution of the identity in our context. The proof of Theorem III.1 then follows Remark III.5.

Proposition III.2 For any $\beta>0$, the trace

$$
\operatorname{Tr} e^{-\beta\left(H_{0}(\mathrm{~h}, X)+z \mathrm{~V}(v, X)-\mu N\right)}
$$

on the Fock space $\mathcal{B}(X)$ is a holomorphic function of $(z, \mu)$ on $\{z \in \mathbb{C} \mid \operatorname{Re} z>0\} \times \mathbb{C}$

Proof: Suppressing h, $v$ and $X$,

$$
\operatorname{Tr} e^{-\beta\left(H_{0}+z \mathrm{~V}-\mu N\right)}=\sum_{n \geq 0} \operatorname{Tr}_{n} e^{-\beta\left(H_{0}+z \mathrm{~V}-\mu N\right)}=\sum_{n \geq 0} e^{\beta \mu n} \operatorname{Tr}_{n} e^{-\beta\left(H_{0}+z \mathrm{~V}\right)}
$$


where $\operatorname{Tr}_{n}$ denotes the trace on the $n$ particle space $\mathcal{B}_{n}$. When restricted to $\mathcal{B}_{n}$, the Hamiltonian $H_{0}+z \mathrm{~V}$ is an operator on a finite dimensional vector space. Therefore, each term $\operatorname{Tr}_{n} e^{-\beta\left(H_{0}+z \mathrm{~V}\right)}$ is an entire function of $(z, \mu)$ on $\mathbb{C}^{2}$. For each $n \geq 0$,

$$
\left|e^{\beta \mu n} \operatorname{Tr}_{n} e^{-\beta\left(H_{0}+z \mathrm{~V}\right)}\right| \leq e^{\beta|\operatorname{Re} \mu| n} \operatorname{dim} \mathcal{B}_{n}(X)\left\|e^{-\beta\left(H_{0}+z \mathrm{~V}\right)}\right\|_{\mathcal{B}_{n}}
$$

where $\|\cdot\|_{\mathcal{B}_{n}}$ is the operator norm on $\mathcal{B}_{n}(X)$. By the Trotter product formula,

$$
\begin{aligned}
\left\|e^{-\beta\left(H_{0}+z \mathrm{~V}\right)}\right\|_{\mathcal{B}_{n}} & =\lim _{m \rightarrow \infty}\left\|\left(e^{-\frac{\beta}{m}\left(H_{0}+\operatorname{Re} z \mathrm{~V}\right)} e^{-\imath \frac{\beta}{m} \operatorname{Im} z \mathrm{~V}}\right)^{m}\right\|_{\mathcal{B}_{n}} \\
& \leq \lim _{m \rightarrow \infty}\left\|e^{-\frac{\beta}{m}\left(H_{0}+\operatorname{Re} z \mathrm{~V}\right)}\right\|_{\mathcal{B}_{n}}^{m}\left\|e^{-\imath \frac{\beta}{m} \operatorname{Im} z \mathrm{~V}}\right\|_{\mathcal{B}_{n}}^{m} \\
& =\lim _{m \rightarrow \infty}\left\|e^{-\frac{\beta}{m}\left(H_{0}+\operatorname{Re} z \mathrm{~V}\right)}\right\|_{\mathcal{B}_{n}}^{m}
\end{aligned}
$$

By Proposition II.17,

$$
\left\|e^{-\frac{\beta}{m}\left(H_{0}+\operatorname{Re} z \mathrm{~V}\right)}\right\|_{\mathcal{B}_{n}}^{m} \leq e^{-\beta\left[\lambda_{0}^{\prime} n+\frac{1}{2} \operatorname{Re} z\left(\frac{\lambda_{0}}{|X|} n^{2}-v_{0} n\right)\right]}
$$

with the $\lambda_{0}=\lambda_{0}(v)$ of Definition II.16. Since $\operatorname{dim} \mathcal{B}_{n}(X) \leq|X|^{n}$, the sum in (III.1) is absolutely convergent, uniformly for $(z, \mu)$ in compact subsets of $\{z \in \mathbb{C} \mid \operatorname{Re} z>0\} \times \mathbb{C}$. This gives the desired analyticity.

Remark III.3 Observe that

$$
\operatorname{Tr} e^{-\beta\left(H_{0}(\mathrm{~h}, X)+z \mathrm{~V}(v, X)-\mu N\right)}=\infty
$$

when $z=0$ and $\mu$ is strictly bigger than the smallest eigenvalue of $h$. This indicates that the "free" limit $z \searrow 0$ is extremely singular.

Lemma III.4 Let $\beta>0$ and let $K$ be any self adjoint operator on $\mathcal{B}(X)$ that commutes with $N$ and obeys

$$
K \geq a\left(\frac{N}{|X|}-\nu\right) N
$$

for some constants $a>0$ and $\nu$. Also, let $\mathrm{R}$ be any map from $\mathbb{N}$ to $(0, \infty)$ such that

$$
\lim _{p \rightarrow \infty} p e^{-\frac{1}{2} \mathrm{R}(p)^{2}}=0
$$

Then,

$$
\begin{aligned}
\operatorname{Tr} e^{-\beta K} & =\lim _{p \rightarrow \infty} \operatorname{Tr}\left(e^{-\frac{\beta}{p} K} \mathrm{I}_{\mathrm{R}(p)}\right)^{p-1} e^{-\frac{\beta}{p} K} \\
& =\lim _{p \rightarrow \infty} \operatorname{Tr} \overbrace{e^{-\frac{\beta}{p} K} \mathrm{I}_{\mathrm{R}(p)} e^{-\frac{\beta}{p} K} \mathrm{I}_{\mathrm{R}(p)} \cdots \mathrm{I}_{\mathrm{R}(p)} e^{-\frac{\beta}{p} K}}^{p \text { exponentials } \mathrm{e}^{-\frac{\beta}{\mathrm{p}} \mathrm{K}}}
\end{aligned}
$$


and

$$
\left\langle\phi\left|e^{-\beta K}\right| \phi^{\prime}\right\rangle=\lim _{p \rightarrow \infty}\left\langle\phi\left|\left(e^{-\frac{\beta}{p} K} \mathrm{I}_{\mathrm{R}(p)}\right)^{p-1} e^{-\frac{\beta}{p} K}\right| \phi^{\prime}\right\rangle
$$

for all $\phi, \phi^{\prime} \in L^{2}(X)$. Furthermore, for each $0<\eta<1$, there is a constant $C_{\eta}$, depending on $\eta, \beta$, a and $\nu$, but independent of $\phi, \phi^{\prime} \in L^{2}(X), p \in \mathbb{N}$ and $X$ such that

$$
\left|\left\langle\phi\left|\left(e^{-\frac{\beta}{p} K} \mathrm{I}_{r}\right)^{p-1} e^{-\frac{\beta}{p} K}\right| \phi^{\prime}\right\rangle\right| \leq e^{\frac{\eta}{2}\left(\|\phi\|^{2}+\left\|\phi^{\prime}\right\|^{2}\right)} e^{C_{\eta}|X|}
$$

Proof: Introduce the local notation

$$
A_{i}=\left\{\begin{array}{ll}
e^{-\frac{\beta}{p} K} & \text { if } i \text { is odd } \\
\mathrm{I}_{\mathrm{R}(p)} & \text { if } i \text { is even }
\end{array}\right\} \quad B_{i}=\left\{\begin{array}{ll}
e^{-\frac{\beta}{p} K} & \text { if } i \text { is odd } \\
\mathbb{1} & \text { if } i \text { is even }
\end{array}\right\}
$$

so that

$$
\left(e^{-\frac{\beta}{p} K} \mathrm{I}_{\mathrm{R}(p)}\right)^{p-1} e^{-\frac{\beta}{p} K}=\prod_{i=1}^{2 p-1} A_{i} \quad \text { and } \quad e^{-\beta K}=\prod_{i=1}^{2 p-1} B_{i}
$$

For any $n \in \mathbb{N}$,

$$
\begin{aligned}
\left|\operatorname{Tr}\left(\prod_{i=1}^{2 p-1} A_{i}-e^{-\beta K}\right)\right| \\
\leq\left|\operatorname{Tr}\left(\prod_{i=1}^{2 p-1} A_{i}-e^{-\beta K}\right) \mathrm{P}_{n}\right|+\left|\operatorname{Tr} \prod_{i=1}^{2 p-1} A_{i}\left(\mathbb{1}-\mathrm{P}_{n}\right)\right|+\left|\operatorname{Tr} e^{-\beta K}\left(\mathbb{1}-\mathrm{P}_{n}\right)\right| \\
=\left|\operatorname{Tr}\left(\prod_{i=1}^{2 p-1} A_{i}-\prod_{i=1}^{2 p-1} B_{i}\right) \mathrm{P}_{n}\right| \\
\quad+\left|\operatorname{Tr} \prod_{i=1}^{2 p-1} A_{i}\left(\mathbb{1}-\mathrm{P}_{n}\right)\right|+\left|\operatorname{Tr} e^{-\beta K}\left(\mathbb{1}-\mathrm{P}_{n}\right)\right|
\end{aligned}
$$

Consider the first line, (III.2). Since $\left\|\mathrm{I}_{\mathrm{R}(p)}\right\| \leq 1$, by part (c) of Theorem II.26, and

$$
K \geq \frac{a}{|X|}(N-\nu|X|) N \geq-\frac{a}{4}|X| \nu^{2} \equiv-K_{0}
$$

we have

$$
\left\|A_{i}\right\|,\left\|B_{i}\right\| \leq \begin{cases}e^{\frac{\beta}{p} K_{0}} & \text { if } i \text { is odd } \\ 1 & \text { if } i \text { is even }\end{cases}
$$

Since $A_{q}-B_{q}=\mathrm{I}_{\mathrm{R}(p)}-\mathbb{1}$ for $i=2,4, \cdots, 2 p-2$ and is zero otherwise, we have, for all $n, p \in \mathbb{N}$,

$$
\begin{aligned}
\left\|\left(\prod_{i=1}^{2 p-1} A_{i}-\prod_{i=1}^{2 p-1} B_{i}\right) \mathrm{P}_{n}\right\| & \leq \sum_{q=1}^{2 p-1}\left\|\prod_{i=1}^{q-1} A_{i}\left(A_{q}-B_{q}\right) \prod_{i=q+1}^{2 p-1} B_{i} \mathrm{P}_{n}\right\| \\
& \leq(p-1) e^{K_{0} \beta}\left\|\left(\mathrm{I}_{\mathrm{R}(p)}-11\right) \mathrm{P}_{n}\right\| \\
& \leq p e^{K_{0} \beta}|X| 2^{n+1} e^{-\mathrm{R}(p)^{2} / 2}
\end{aligned}
$$


Consequently,

$$
\begin{aligned}
& \left|\operatorname{Tr}\left(\left(e^{-\frac{\beta}{p} K} \mathrm{I}_{\mathrm{R}(p)}\right)^{p-1} e^{-\frac{\beta}{p} K}-e^{-\beta K}\right) \mathrm{P}_{n}\right| \\
& \leq p e^{K_{0} \beta}|X| 2^{n+1} e^{-\mathrm{R}(p)^{2} / 2} \operatorname{Tr} \mathrm{P}_{n} \\
& \leq p e^{K_{0} \beta}|X| 2^{n+1} e^{-\mathrm{R}(p)^{2} / 2} \frac{(n+|X|) !}{n !|X| !}
\end{aligned}
$$

Hence, for any fixed $n \in \mathbb{N}$,

$$
\lim _{p \rightarrow \infty}\left|\operatorname{Tr}\left(\left(e^{-\frac{\beta}{p} K} \mathrm{I}_{\mathrm{R}(p)}\right)^{p-1} e^{-\frac{\beta}{p} K}-e^{-\beta K}\right) \mathrm{P}_{n}\right|=0
$$

Now we consider the second line, (III.3). For all $m \geq 1,\left.K\right|_{\mathcal{B}_{m}} \geq a\left(\frac{m}{|X|}-\nu\right) m$ and

$$
\left\|\left.\prod_{i=1}^{2 p-1} A_{i}\right|_{\mathcal{B}_{m}}\right\|, \quad\left\|\left.e^{-\beta K}\right|_{\mathcal{B}_{m}}\right\| \leq e^{-\beta a\left(\frac{m}{|X|}-\nu\right) m}
$$

and it follows that

$$
\left|\operatorname{Tr} \prod_{i=1}^{2 p-1} A_{i}\right|_{\mathcal{B}_{m}}\left|, \quad \operatorname{Tr} e^{-\beta K}\right|_{\mathcal{B}_{m}} \leq e^{-\beta a\left(\frac{m}{|X|}-\nu\right) m} \frac{(m+|X|-1) !}{m !(|X|-1) !}
$$

If we impose the stronger condition $m \geq n$ with $\frac{n}{|X|} \geq 2 \nu$, the last inequality becomes

$$
\left|\operatorname{Tr} \prod_{i=1}^{2 p-1} A_{i}\right|_{\mathcal{B}_{m}}\left|, \quad \operatorname{Tr} e^{-\beta K}\right|_{\mathcal{B}_{m}} \leq e^{-\frac{a}{2|X|} m^{2} \beta} \frac{(m+|X|-1) !}{m !(|X|-1) !}
$$

and

$$
\frac{(m+|X|-1) !}{m !(|X|-1) !} \leq \frac{(m+|X|-1)^{|X|-1}}{(|X|-1) !} \leq \frac{(c m)^{|X|-1}}{(|X|-1) !}
$$

where, $c=\frac{1}{2 \nu}+1$. Now, we have, if $n \geq 2|X| \nu$,

$$
\begin{aligned}
\left|\operatorname{Tr} \prod_{i=1}^{2 p-1} A_{i}\left(\mathbb{1}-\mathrm{P}_{n}\right)\right|, & \operatorname{Tr} e^{-\beta K}\left(\mathbb{1}-\mathrm{P}_{n}\right) \leq \sum_{m>n} e^{-\frac{a}{2|X|} m^{2} \beta} \frac{(c m)^{|X|-1}}{(|X|-1) !} \\
& \leq \int_{n}^{\infty} d t e^{-\frac{a}{2|X|} t^{2} \beta \frac{(c t \mid)|X|-1}{(|X|-1) !}} \\
& \leq e^{-\frac{a}{4|X|} n^{2} \beta} \int_{0}^{\infty} d t e^{-\frac{a}{4|X|} t^{2} \beta \frac{(c t)^{|X|-1}}{(|X|-1) !}} \\
& \leq C e^{-\frac{a}{4|X|} n^{2} \beta}
\end{aligned}
$$

for some positive constant $C$, depending only on $\beta, a, \nu$ and $|X|$. The first claim now follows from the observation that this converges to zero as $n \rightarrow \infty$, uniformly in $\mathrm{R}(p)$ and $p$. In fact this proves convergence in trace norm and hence convergence in operator norm and also weak convergence, so that this also proves the second claim. 
Finally, we prove the bound. By (III.4) and Proposition II.22,

$$
\begin{aligned}
\left|\left\langle\phi\left|\left(e^{-\frac{\beta}{p} K} \mathrm{I}_{R}\right)^{p-1} e^{-\frac{\beta}{p} K}\right| \phi^{\prime}\right\rangle\right| & \leq \sum_{m=0}^{\infty} \frac{\|\phi\|^{m}}{\sqrt{m !}} e^{-\beta a\left(\frac{m}{|X|}-\nu\right) m} \frac{\left\|\phi^{\prime}\right\|^{m}}{\sqrt{m !}} \\
& \leq \sum_{m=0}^{\infty} \frac{t^{m}}{m !} e^{-\beta a\left(\frac{m}{|X|}-\nu\right) m}
\end{aligned}
$$

where $t=\frac{1}{2}\left(\|\phi\|^{2}+\left\|\phi^{\prime}\right\|^{2}\right)$. Observe that, for any $\gamma>0$,

$$
\begin{aligned}
a \beta\left(\frac{m^{2}}{|X|}-\nu m-\gamma m\right) & =\frac{a \beta}{|X|}\left(m-\frac{\nu+\gamma}{2}|X|\right)^{2}-\frac{a}{4} \beta(\nu+\gamma)^{2}|X| \\
& \geq-\frac{a \beta}{4}(\nu+\gamma)^{2}|X|
\end{aligned}
$$

Thus

$$
\begin{aligned}
\left|\left\langle\phi\left|\left(e^{-\frac{\beta}{p} K} \mathrm{I}_{R}\right)^{p-1} e^{-\frac{\beta}{p} K}\right| \phi^{\prime}\right\rangle\right| & \leq \sum_{m=0}^{\infty} \frac{t^{m}}{m !} e^{-a \beta \gamma m} e^{-a \beta\left(\frac{m^{2}}{|X|}-\nu m-\gamma m\right)} \\
& \leq \sum_{m=0}^{\infty} \frac{t^{m}}{m !} e^{-a \beta \gamma m} e^{\frac{a \beta}{4}(\nu+\gamma)^{2}|X|} \\
& =e^{e^{-a \beta \gamma} t} e^{\frac{a \beta}{4}(\nu+\gamma)^{2}|X|}
\end{aligned}
$$

It now suffices to choose $\gamma$ so that $\eta=e^{-a \beta \gamma}$ and then set $C_{\eta}=\frac{a \beta}{4}(\nu+\gamma)^{2}$.

Remark III.5 If $\mathrm{R}(p) \geq c|\ln p|^{\frac{1}{2}+\varepsilon}$, then $\lim _{p \rightarrow \infty} p e^{-\mathrm{R}(p)^{2}}=0$. Also if $\mathrm{R}(p) \geq c|\ln p|^{\frac{1}{2}}$ with $c>1$, then $\lim _{p \rightarrow \infty} p e^{-\mathrm{R}(p)^{2}}=0$.

Proof of Theorem III.1: By (III.6), the strong convergence of $\mathrm{I}_{r}$ to 1 , and Proposition II.28.a

$$
\operatorname{Tr} e^{-\beta K}=\lim _{p \rightarrow \infty} \operatorname{Tr} e^{-\beta K} \mathrm{I}_{\mathrm{R}(p)}=\lim _{p \rightarrow \infty} \int d \mu_{\mathrm{R}(p)}\left(\phi_{0}^{*}, \phi_{0}\right) e^{-\int d \mathbf{y}\left|\phi_{0}(\mathbf{y})\right|^{2}}\left\langle\phi_{0}\left|e^{-\beta K}\right| \phi_{0}\right\rangle
$$

It follows from Lemma III.4 and the dominated convergence theorem that

$$
\operatorname{Tr} e^{-\beta K}=\lim _{p \rightarrow \infty} \int \prod_{\tau \in \mathcal{T}_{p}}\left[d \mu_{\mathrm{R}(p)}\left(\phi_{\tau}^{*}, \phi_{\tau}\right) e^{-\int d \mathbf{y}\left|\phi_{\tau}(\mathbf{y})\right|^{2}}\right] \prod_{\tau \in \mathcal{T}_{p}}\left\langle\phi_{\tau-\varepsilon}\left|e^{-\varepsilon K}\right| \phi_{\tau}\right\rangle
$$

as desired.

The logarithm of $\left\langle\alpha\left|e^{-\varepsilon K}\right| \phi\right\rangle$

Theorem III.1 is a rigorous version of the intermediate representation (I.5). As discussed in the introduction, it now remains to show that one may replace $\left\langle\alpha\left|e^{-\varepsilon K}\right| \phi\right\rangle$ by $e^{\int d \mathbf{y} \alpha(\mathbf{y})^{*} \phi(\mathbf{y})-\varepsilon K\left(\alpha^{*}, \phi\right)}$ in the formula for $\operatorname{Tr} e^{-\beta K}$ of Theorem III.1. In Theorem III.13, 
below, we show that this is indeed the case, provided $\mathrm{R}(p)$ is chosen appropriately. To prepare for that, we explicitly find the $\log$ arithm $F\left(\varepsilon, \alpha^{*}, \phi\right)=\ln \left\langle\alpha\left|e^{-\varepsilon K}\right| \phi\right\rangle$ at least for $\alpha$ and $\phi$ not too large, and show that

$$
F\left(\varepsilon, \alpha^{*}, \phi\right)=\int_{X} d \mathbf{x} \alpha(\mathbf{x})^{*} \phi(\mathbf{x})-\varepsilon K\left(\alpha^{*}, \phi\right)+O\left(\varepsilon^{2}\right)
$$

This expression is the same, to order $\varepsilon$, as

$$
\begin{aligned}
F\left(\varepsilon, \alpha^{*}, \phi\right)=\iint_{X^{2}} d \mathbf{x} d \mathbf{y} \alpha & (\mathbf{x})^{*} j(\varepsilon ; \mathbf{x}, \mathbf{y}) \phi(\mathbf{y}) \\
& -\frac{1}{2} \varepsilon \iint_{X^{2}} d \mathbf{x} d \mathbf{y} \alpha(\mathbf{x})^{*} \alpha(\mathbf{y})^{*} v(\mathbf{x}, \mathbf{y}) \phi(\mathbf{x}) \phi(\mathbf{y})+O\left(\varepsilon^{2}\right)
\end{aligned}
$$

provided $j(\varepsilon)=\mathbb{1}-\varepsilon(\mathrm{h}-\mu)+O\left(\varepsilon^{2}\right)$. For application of renormalization group methods, the latter form is more convenient. So we show it too. We typically use the supremum norm

$$
|\phi|_{X}=\max _{\mathbf{x} \in X}|\phi(\mathbf{x})|
$$

to measure the size of the field $\phi$ and the norm

$$
\|\mathrm{h}\|_{1, \infty}=\max _{\mathbf{x} \in X} \int_{X} d \mathbf{y}|\mathrm{h}(\mathbf{x}, \mathbf{y})|
$$

to measure the size of (symmetric) integral operators on $L^{2}(X)$.

Proposition III.6 For each $\varepsilon>0$, there is an analytic function $F\left(\varepsilon, \alpha^{*}, \phi\right)$ such that

$$
\left\langle\alpha\left|e^{-\varepsilon K}\right| \phi\right\rangle=e^{F\left(\varepsilon, \alpha^{*}, \phi\right)}
$$

on the domain $|\alpha|_{X},|\phi|_{X}<C_{\varepsilon}$ where $C_{\varepsilon}=\left[8 e^{\varepsilon\left(\|\mathrm{h}\|_{1, \infty}+\mu+v_{0}\right)} \varepsilon\|v\|_{1, \infty}\right]^{-1 / 2}$ with $v_{0}=$ $\max _{\mathbf{x} \in X} v(\mathbf{x}, \mathbf{x})$.

Let $c_{j}>0$ and $j(\varepsilon ; \mathbf{x}, \mathbf{y})$ be the kernel of an operator obeying

$$
\left\|j(\varepsilon)-e^{-\varepsilon(\mathrm{h}-\mu)}\right\|_{1, \infty} \leq c_{j} \varepsilon^{2}
$$

Define the function $\mathcal{F}_{1}\left(\varepsilon, \alpha^{*}, \phi\right)$ by

$$
\begin{aligned}
F\left(\varepsilon, \alpha^{*}, \phi\right)=\iint_{X^{2}} d \mathbf{x} d \mathbf{y} \alpha(\mathbf{x})^{*} j(\varepsilon ; \mathbf{x}, \mathbf{y}) \phi(\mathbf{y}) \\
\quad-\frac{\varepsilon}{2} \iint_{X^{2}} d \mathbf{x} d \mathbf{y} \alpha(\mathbf{x})^{*} \alpha(\mathbf{y})^{*} v(\mathbf{x}, \mathbf{y}) \phi(\mathbf{x}) \phi(\mathbf{y})+\mathcal{F}_{1}\left(\varepsilon, \alpha^{*}, \phi\right)
\end{aligned}
$$

For every $\varepsilon_{0}>0$ there is a constant const (depending only on $\varepsilon_{0},\|\mathrm{~h}\|_{1, \infty}, v, c_{j}$ and $\mu$ ) such that for all $0<\varepsilon<\varepsilon_{0}$

$$
\left|\mathcal{F}_{1}\left(\varepsilon, \alpha^{*}, \phi\right)\right| \leq \operatorname{const} \varepsilon^{2}\left(R^{2}+\|v\|_{1, \infty}^{2} R^{6}\right)|X|
$$

for all $|\alpha|_{X},|\phi|_{X} \leq R \leq \frac{1}{2} C_{\varepsilon}$.

An immediate consequence is 
Corollary III.7 We use the notation of Proposition III.6. Define the function $\mathcal{F}_{0}\left(\varepsilon, \alpha^{*}, \phi\right)$ by

$$
F\left(\varepsilon, \alpha^{*}, \phi\right)=\int_{X} d \mathbf{x} \alpha(\mathbf{x})^{*} \phi(\mathbf{x})-\varepsilon K\left(\alpha^{*}, \phi\right)+\mathcal{F}_{0}\left(\varepsilon, \alpha^{*}, \phi\right)
$$

where

$$
\begin{aligned}
K\left(\alpha^{*}, \phi\right)=\iint_{X^{2}} d \mathbf{x} d \mathbf{y} \alpha & (\mathbf{x})^{*} \mathrm{~h}(\mathbf{x}, \mathbf{y}) \phi(\mathbf{y})-\mu \int_{X} d \mathbf{x} \alpha(\mathbf{x})^{*} \phi(\mathbf{x}) \\
& +\frac{1}{2} \iint_{X^{2}} d \mathbf{x} d \mathbf{y} \alpha(\mathbf{x})^{*} \alpha(\mathbf{y})^{*} v(\mathbf{x}, \mathbf{y}) \phi(\mathbf{x}) \phi(\mathbf{y})
\end{aligned}
$$

For every $\varepsilon_{0}>0$ there is a constant const (depending only on $\varepsilon_{0},\|\mathrm{~h}\|_{1, \infty}, v$ and $\mu$ ) such that for all $0<\varepsilon<\varepsilon_{0}$

$$
\left|\mathcal{F}_{0}\left(\varepsilon, \alpha^{*}, \phi\right)\right| \leq \operatorname{const} \varepsilon^{2}\left(R^{2}+\|v\|_{1, \infty}^{2} R^{6}\right)|X|
$$

for all $|\alpha|_{X},|\phi|_{X} \leq R \leq \frac{1}{2} C_{\varepsilon}$.

We now prove a number of lemmas leading up to the proof of Proposition III.6, following Lemma III.11.

Lemma III.8 Let $\varepsilon>0$. There exists a function $F\left(\varepsilon, \alpha^{*}, \phi\right)$, analytic in $\alpha^{*}$ and $\phi$ in a neighbourhood of the origin, such that

$$
\left\langle\alpha\left|e^{-\varepsilon K}\right| \phi\right\rangle=e^{F\left(\varepsilon, \alpha^{*}, \phi\right)}
$$

$F$ satisfies the differential equation

$$
\frac{\partial}{\partial \varepsilon} F=-\mathcal{K}\left(\alpha^{*}, \frac{\partial}{\partial \alpha^{*}}\right) F-\frac{1}{2} \iint_{X} d \mathbf{x} d \mathbf{y} \alpha(\mathbf{x})^{*} \alpha(\mathbf{y})^{*} v(\mathbf{x}, \mathbf{y}) \frac{\partial F}{\partial \alpha(\mathbf{x})^{*}} \frac{\partial F}{\partial \alpha(\mathbf{y})^{*}}
$$

with the initial condition

$$
F\left(0, \alpha^{*}, \phi\right)=\int_{X} d \mathbf{x} \alpha(\mathbf{x})^{*} \phi(\mathbf{x})
$$

Here,

$$
\begin{aligned}
\mathcal{K}\left(\alpha^{*}, \frac{\partial}{\partial \alpha^{*}}\right)=\iint_{X} d \mathbf{x} d \mathbf{y} \alpha(\mathbf{x})^{*} \mathrm{~h}(\mathbf{x}, \mathbf{y}) \frac{\partial}{\partial \alpha(\mathbf{y})^{*}}-\mu \int_{X} d \mathbf{x} \alpha(\mathbf{x})^{*} \frac{\partial}{\partial \alpha(\mathbf{x})^{*}} \\
+\frac{1}{2} \iint_{X} d \mathbf{x} d \mathbf{y} \alpha(\mathbf{x})^{*} \alpha(\mathbf{y})^{*} v(\mathbf{x}, \mathbf{y}) \frac{\partial}{\partial \alpha(\mathbf{x})^{*}} \frac{\partial}{\partial \alpha(\mathbf{y})^{*}}
\end{aligned}
$$

Proof: Set $\lambda_{0}=\lambda_{0}(v)$ as in Definition II.16. Since, by Propositions II.22 and II.17,

$$
\begin{aligned}
\left|\left\langle\alpha\left|e^{-\varepsilon K}\right| \phi\right\rangle\right| & \leq \sum_{m=0}^{\infty} \frac{\|\alpha\|^{m}}{\sqrt{m !}}\left\|\left.e^{-\varepsilon K}\right|_{\mathcal{B}_{m}} \mid\right\| \frac{\|\phi\|^{m}}{\sqrt{m !}} \leq \sum_{m=0}^{\infty} \frac{\|\alpha\|^{m}}{\sqrt{m !}} e^{-\varepsilon\left(\frac{\lambda_{0}}{2} \mid \frac{m}{|X|}+\lambda_{0}^{\prime}-\frac{v_{0}}{2}-\mu\right) m} \frac{\|\phi\|^{m}}{\sqrt{m !}} \\
& \leq \sum_{m=0}^{\infty} \frac{1}{2^{m} m !}\left(\|\alpha\|^{2}+\|\phi\|^{2}\right)^{m} e^{-\varepsilon\left(\frac{\lambda_{0}}{2} \frac{m}{|X|}+\lambda_{0}^{\prime}-\frac{v_{0}}{2}-\mu\right) m}
\end{aligned}
$$


the Taylor series expansion of $\left\langle\alpha\left|e^{-\varepsilon K}\right| \phi\right\rangle$ converges for all $\alpha, \phi \in L^{2}(X)$ so that $\left\langle\alpha\left|e^{-\varepsilon K}\right| \phi\right\rangle$ is an entire function of $\alpha^{*}$ and $\phi$. Since $\langle\alpha \mid \phi\rangle=e^{\int \alpha^{*}(\mathbf{x}) \phi(\mathbf{x}) d \mathbf{x}} \neq 0$, the matrix element has the representation

$$
\left\langle\alpha\left|e^{-\varepsilon K}\right| \phi\right\rangle=e^{F\left(\varepsilon, \alpha^{*}, \phi\right)}
$$

in a neighbourhood of 0 , with $F\left(\varepsilon, \alpha^{*}, \phi\right)$ is analytic in $\alpha^{*}, \phi$. We have

$$
\begin{array}{r}
e^{F\left(\varepsilon, \alpha^{*}, \phi\right)} \frac{\partial}{\partial \varepsilon} F=\frac{\partial}{\partial \varepsilon} e^{F\left(\varepsilon, \alpha^{*}, \phi\right)}=\frac{\partial}{\partial \varepsilon}\left\langle\alpha\left|e^{-\varepsilon K}\right| \phi\right\rangle=-\left\langle\alpha\left|K e^{-\varepsilon K}\right| \phi\right\rangle \\
=-\langle\alpha|\left[\iint_{X^{2}} d \mathbf{x} d \mathbf{y} \psi^{\dagger}(\mathbf{x}) \mathrm{h}(\mathbf{x}, \mathbf{y}) \psi(\mathbf{y})-\mu \int_{X} d \mathbf{x} \psi^{\dagger}(\mathbf{x}) \psi(\mathbf{x})\right. \\
\left.+\frac{1}{2} \iint_{X^{2}} d \mathbf{x} d \mathbf{y} \psi^{\dagger}(\mathbf{x}) \psi^{\dagger}(\mathbf{y}) v(\mathbf{x}, \mathbf{y}) \psi(\mathbf{x}) \psi(\mathbf{y})\right] e^{-\varepsilon K}|\phi\rangle
\end{array}
$$

By Proposition II.20, the first term

$$
\begin{aligned}
\langle\alpha|\left[\iint_{X^{2}} d \mathbf{x} d \mathbf{y}\right. & \left.\psi^{\dagger}(\mathbf{x}) \mathrm{h}(\mathbf{x}, \mathbf{y}) \psi(\mathbf{y})\right] e^{-\varepsilon K}|\phi\rangle \\
& =\iint_{X^{2}} d \mathbf{x} d \mathbf{y} \mathrm{h}(\mathbf{x}, \mathbf{y})\left\langle\alpha\left|\psi^{\dagger}(\mathbf{x}) \psi(\mathbf{y}) e^{-\varepsilon K}\right| \phi\right\rangle \\
& =\iint_{X^{2}} d \mathbf{x} d \mathbf{y} \alpha(\mathbf{x})^{*} \mathrm{~h}(\mathbf{x}, \mathbf{y})\left\langle\alpha\left|\psi(\mathbf{y}) e^{-\varepsilon K}\right| \phi\right\rangle \\
& =\iint_{X^{2}} d \mathbf{x} d \mathbf{y} \alpha(\mathbf{x})^{*} \mathrm{~h}(\mathbf{x}, \mathbf{y}) \frac{\partial}{\partial \alpha(\mathbf{y})^{*}}\left\langle\alpha\left|e^{-\varepsilon K}\right| \phi\right\rangle \\
& =\iint_{X^{2}} d \mathbf{x} d \mathbf{y} \alpha(\mathbf{x})^{*} \mathrm{~h}(\mathbf{x}, \mathbf{y}) \frac{\partial}{\partial \alpha(\mathbf{y})^{*}} e^{F\left(\varepsilon, \alpha^{*}, \phi\right)}
\end{aligned}
$$

Treating the other two terms similarly,

$$
\begin{aligned}
e^{F\left(\varepsilon, \alpha^{*}, \phi\right)} \frac{\partial}{\partial \varepsilon} F=-\left[\iint_{X^{2}} d \mathbf{x}\right. & d \mathbf{y} \alpha(\mathbf{x})^{*} \mathrm{~h}(\mathbf{x}, \mathbf{y}) \frac{\partial}{\partial \alpha(\mathbf{y})^{*}}-\mu \int_{X} d \mathbf{x} \alpha(\mathbf{x})^{*} \frac{\partial}{\partial \alpha(\mathbf{x})^{*}} \\
+ & \left.\frac{1}{2} \iint_{X^{2}} d \mathbf{x} d \mathbf{y} \alpha(\mathbf{x})^{*} \alpha(\mathbf{y})^{*} v(\mathbf{x}, \mathbf{y}) \frac{\partial}{\partial \alpha(\mathbf{x})^{*}} \frac{\partial}{\partial \alpha(\mathbf{y})^{*}}\right] e^{F\left(\varepsilon, \alpha^{*}, \phi\right)}
\end{aligned}
$$

The differential equation for $F$ follows.

Lemma III.9 The function $F\left(\varepsilon, \alpha^{*}, \phi\right)$ of Lemma III.8 has an expansion

$$
F\left(\varepsilon, \alpha^{*}, \phi\right)=\sum_{n=1}^{\infty} \int_{X^{2 n}} d^{n} \tilde{\mathbf{x}} d^{n} \tilde{\mathbf{y}} e^{n \mu} F_{n}(\varepsilon, \tilde{\mathbf{x}}, \tilde{\mathbf{y}}) \prod_{i=1}^{n} \alpha\left(\mathbf{x}_{i}\right)^{*} \phi\left(\mathbf{y}_{i}\right) \text { where } \begin{array}{r}
\tilde{\mathbf{x}}=\left(\mathbf{x}_{1}, \cdots, \mathbf{x}_{n}\right) \\
\tilde{\mathbf{y}}=\left(\mathbf{y}_{1}, \cdots, \mathbf{y}_{n}\right)
\end{array}
$$


in powers of the fields $\alpha^{*}$ and $\phi$. Furthermore

$$
\begin{aligned}
& F_{1}\left(\varepsilon, \mathbf{x}_{1}, \mathbf{y}_{1}\right)=e^{-\varepsilon \mathrm{h}}\left(\mathbf{x}_{1}, \mathbf{y}_{1}\right) \\
& F_{n}(\varepsilon, \tilde{\mathbf{x}}, \tilde{\mathbf{y}})=-\frac{1}{2} \int_{0}^{\varepsilon} d \tau \int_{X^{n}} d^{n} \tilde{\mathbf{x}}^{\prime}\left(e^{-(\varepsilon-\tau) \mathrm{H}_{n}}\right)\left(\tilde{\mathbf{x}}, \tilde{\mathbf{x}}^{\prime}\right) \sum_{m=1}^{n-1} S_{\tilde{\mathbf{x}}, \tilde{\mathbf{y}}} \sum_{j=1}^{m} \sum_{k=m+1}^{n} v\left(\mathbf{x}_{j}^{\prime}, \mathbf{x}_{k}^{\prime}\right) \\
& F_{m}\left(\tau, \tilde{\mathbf{x}}_{[\leq m]}^{\prime}, \tilde{\mathbf{y}}_{[\leq m]}\right) F_{n-m}\left(\tau, \tilde{\mathbf{x}}_{[>m]}^{\prime}, \tilde{\mathbf{y}}_{[>m]}\right)
\end{aligned}
$$

where $S_{\tilde{\mathbf{x}}, \tilde{\mathbf{y}}}$ denotes independent symmetrization in the $\mathbf{x}$ variables and the $\mathbf{y}$ variables,

$$
\mathrm{H}_{n}\left(\tilde{\mathbf{x}}, \tilde{\mathbf{x}}^{\prime}\right)=\sum_{k=1}^{n} \mathrm{~h}\left(\mathbf{x}_{k}, \mathbf{x}_{k}^{\prime}\right) \prod_{\substack{1 \leq \ell \leq n \\ \ell \neq k}} \delta_{\mathbf{x}_{\ell}}\left(\mathbf{x}_{\ell}^{\prime}\right)+\frac{1}{2} \sum_{\substack{j, k=1 \\ j \neq k}}^{n} v\left(\mathbf{x}_{j}, \mathbf{x}_{k}\right) \prod_{1 \leq \ell \leq n} \delta_{\mathbf{x}_{\ell}}\left(\mathbf{x}_{\ell}^{\prime}\right)
$$

and

$$
\tilde{\mathbf{x}}^{\prime}=\left(\mathbf{x}_{1}^{\prime}, \cdots, \mathbf{x}_{n}^{\prime}\right) \quad \tilde{\mathbf{x}}_{[\leq m]}=\left(\mathbf{x}_{1}, \cdots, \mathbf{x}_{m}\right) \quad \text { and } \quad \tilde{\mathbf{x}}_{[>m]}=\left(\mathbf{x}_{m+1}, \cdots, \mathbf{x}_{n}\right)
$$

Proof: $\quad$ Expand $F$ in the power series

$$
F\left(\varepsilon, \alpha^{*}, \phi\right)=\sum_{n=1}^{\infty} \int d^{n} \tilde{\mathbf{x}} e^{n \varepsilon \mu} F_{n}(\varepsilon, \tilde{\mathbf{x}}, \phi) \prod_{i=1}^{n} \alpha\left(\mathbf{x}_{i}\right)^{*}
$$

in $\alpha^{*}$ with coefficients $F_{n}(\varepsilon, \tilde{\mathbf{x}}, \phi)$ that are symmetric under permutation of the $\mathbf{x}_{k}$ 's. The constant term is zero because $\left.\left\langle\alpha\left|e^{-\varepsilon K}\right| \phi\right\rangle\right|_{\alpha=\phi=0}=1$. Each $F_{n}(\varepsilon, \tilde{\mathbf{x}}, \phi)$ has degree $n$ in $\phi$ because the fact that $e^{-\varepsilon K}$ preserves particle number implies that $F\left(\varepsilon, e^{-i \theta} \alpha^{*}, e^{i \theta} \phi\right)=$ $F\left(\varepsilon, \alpha^{*}, \phi\right)$ for all real $\theta$. Observe that

$$
e^{n \varepsilon \mu} F_{n}(\varepsilon, \tilde{\mathbf{x}}, \phi)=\left.\frac{1}{n !} \frac{\partial}{\partial \alpha\left(\mathbf{x}_{1}\right)^{*}} \cdots \frac{\partial}{\partial \alpha\left(\mathbf{x}_{n}\right)^{*}} F\left(\varepsilon, \alpha^{*}, \phi\right)\right|_{\alpha=0}
$$

and

$$
\begin{aligned}
\frac{1}{n !} \frac{\partial}{\partial \alpha\left(\mathbf{x}_{1}\right)^{*}} \cdots \frac{\partial}{\partial \alpha\left(\mathbf{x}_{n}\right)^{*}} & \left.\iint_{X^{2}} d \mathbf{x} d \mathbf{y} \alpha(\mathbf{x})^{*} \mathrm{~h}(\mathbf{x}, \mathbf{y}) \frac{\partial}{\partial \alpha(\mathbf{y})^{*}} F\left(\varepsilon, \alpha^{*}, \phi\right)\right|_{\alpha=0} \\
& =\left.\sum_{k=1}^{n} \frac{1}{n !} \int_{X} d \mathbf{y} \mathrm{h}\left(\mathbf{x}_{k}, \mathbf{y}\right) \frac{\partial}{\partial \alpha(\mathbf{y})^{*}} \prod_{\ell \neq k} \frac{\partial}{\partial \alpha\left(\mathbf{x}_{\ell}\right)^{*}} F\left(\varepsilon, \alpha^{*}, \phi\right)\right|_{\alpha=0} \\
& =\sum_{k=1}^{n} \frac{1}{n !} \int_{X} d \mathbf{x}_{k}^{\prime} \mathrm{h}\left(\mathbf{x}_{k}, \mathbf{x}_{k}^{\prime}\right) e^{n \varepsilon \mu} F_{n}\left(\varepsilon, \mathbf{x}_{1}, \cdots, \mathbf{x}_{k-1}, \mathbf{x}_{k}^{\prime}, \mathbf{x}_{k+1}, \cdots, \mathbf{x}_{n}, \phi\right)
\end{aligned}
$$

and

$$
\begin{aligned}
& \frac{1}{n !} \frac{\partial}{\partial \alpha\left(\mathbf{x}_{1}\right)^{*}} \cdots \frac{\partial}{\partial \alpha\left(\mathbf{x}_{n}\right)^{*}} G\left(\alpha^{*}\right) H\left(\alpha^{*}\right) \\
& =\sum_{m=0}^{n} S\left[\frac{1}{m !} \prod_{\ell=1}^{m} \frac{\partial}{\partial \alpha\left(\mathbf{x}_{\ell}\right)^{*}} G\left(\alpha^{*}\right)\right]\left[\frac{1}{(n-m) !} \prod_{\ell=m+1}^{n} \frac{\partial}{\partial \alpha\left(\mathbf{x}_{\ell}\right)^{*}} H\left(\alpha^{*}\right)\right]
\end{aligned}
$$


where $S$ denotes the symmetrization operator in the variables $\mathbf{x}_{k}$. Thus Lemma III.8 gives the system of equations

$$
\begin{aligned}
& \frac{\partial}{\partial \varepsilon} F_{1}\left(\varepsilon, \mathbf{x}_{1}, \phi\right)=-\int d \mathbf{x}_{1}^{\prime} \mathrm{h}\left(\mathbf{x}_{1}, \mathbf{x}_{1}^{\prime}\right) F_{1}\left(\varepsilon, \mathbf{x}_{1}^{\prime}, \phi\right) \quad \text { and for } n>1 \\
& \frac{\partial}{\partial \varepsilon} F_{n}(\varepsilon, \tilde{\mathbf{x}}, \phi)=-\int d^{n} \tilde{\mathbf{x}}^{\prime} \mathrm{H}_{n}\left(\tilde{\mathbf{x}}, \tilde{\mathbf{x}}^{\prime}\right) F_{n}\left(\varepsilon, \tilde{\mathbf{x}}^{\prime}, \phi\right) \\
& -\frac{1}{2} \sum_{m=1}^{n-1} S \sum_{j=1}^{m} \sum_{k=m+1}^{n} v\left(\mathbf{x}_{j}, \mathbf{x}_{k}\right) F_{m}\left(\varepsilon, \mathbf{x}_{1}, \cdots, \mathbf{x}_{m}, \phi\right) F_{n-m}\left(\varepsilon, \mathbf{x}_{m+1}, \cdots, \mathbf{x}_{n}, \phi\right)
\end{aligned}
$$

with the initial condition

$$
F_{1}\left(0, \mathbf{x}_{1}, \phi\right)=\phi\left(\mathbf{x}_{1}\right), \quad F_{n}(0, \tilde{\mathbf{x}}, \phi)=0 \text { for } n>1
$$

The "integral" form of these equations is

$$
\begin{aligned}
F_{1}\left(\varepsilon, \mathbf{x}_{1}, \phi\right) & =\left(e^{-\varepsilon \mathrm{h}} \phi\right)\left(\mathbf{x}_{1}\right) \\
F_{n}(\varepsilon, \tilde{\mathbf{x}}, \phi) & =-\frac{1}{2} \int_{0}^{\varepsilon} d \tau \int d \tilde{\mathbf{x}}^{\prime}\left(e^{-(\varepsilon-\tau) \mathrm{H}_{n}}\right)\left(\tilde{\mathbf{x}}, \tilde{\mathbf{x}}^{\prime}\right) \sum_{m=1}^{n-1} S \sum_{j=1}^{m} \sum_{k=m+1}^{n} v\left(\mathbf{x}_{j}^{\prime}, \mathbf{x}_{k}^{\prime}\right) \\
& F_{m}\left(\tau, \mathbf{x}_{1}^{\prime}, \cdots, \mathbf{x}_{m}^{\prime}, \phi\right) F_{n-m}\left(\tau, \mathbf{x}_{m+1}^{\prime}, \cdots, \mathbf{x}_{n}^{\prime}, \phi\right)
\end{aligned}
$$

We remark that the fact that $F_{n}(\varepsilon, \tilde{\mathbf{x}}, \phi)$ is of degree $n$ in $\phi$ also follows by induction on $n$ from these equations. Writing

$$
F_{n}(\varepsilon, \tilde{\mathbf{x}}, \phi)=\int d \tilde{\mathbf{y}} F_{n}(\varepsilon, \tilde{\mathbf{x}}, \tilde{\mathbf{y}}) \prod_{i=1}^{n} \phi\left(\mathbf{y}_{i}\right)
$$

for each $n \geq 1$, with the $F_{n}(\varepsilon, \tilde{\mathbf{x}}, \tilde{\mathbf{y}})$ 's symmetric under permutations of the $\mathbf{y}_{k}$ 's too, defines the functions of the Lemma.

We now estimate the norm of the operator $e^{-\varepsilon \mathrm{H}_{n}}$ of Lemma III.9, acting on functions $F: X^{n} \times X^{n} \rightarrow \mathbb{C}$. The space of functions is equipped with the norm

$$
\|F\|_{1, \infty}=\max _{1 \leq j \leq 2 n} \max _{\mathbf{x}_{j} \in X} \int_{X^{2 n-1}}\left|F\left(\mathbf{x}_{1}, \cdots, \mathbf{x}_{2 n}\right)\right| \prod_{\substack{1 \leq i \leq 2 n \\ i \neq j}} d \mathbf{x}_{i}
$$

Lemma III.10 Write

$$
\mathrm{H}_{n}\left(\tilde{\mathbf{x}}, \tilde{\mathbf{x}}^{\prime}\right)=\mathrm{h}_{n}\left(\tilde{\mathbf{x}}, \tilde{\mathbf{x}}^{\prime}\right)+V_{n}\left(\tilde{\mathbf{x}}, \tilde{\mathbf{x}}^{\prime}\right)
$$

where

$$
\begin{aligned}
& \mathrm{h}_{n}\left(\tilde{\mathbf{x}}, \tilde{\mathbf{x}}^{\prime}\right)=\sum_{k=1}^{n} \mathrm{~h}\left(\mathbf{x}_{k}, \mathbf{x}_{k}^{\prime}\right) \prod_{\substack{1 \leq \ell \leq n \\
\ell \neq k}} \delta_{\mathbf{x}_{\ell}}\left(\mathbf{x}_{\ell}^{\prime}\right) \\
& V_{n}\left(\tilde{\mathbf{x}}, \tilde{\mathbf{x}}^{\prime}\right)=\frac{1}{2} \sum_{\substack{j, k=1 \\
j \neq k}}^{n} v\left(\mathbf{x}_{j}, \mathbf{x}_{k}\right) \prod_{\substack{1 \leq \ell \leq n \\
\mathbf{x}_{\ell}}} \delta_{\left.\mathbf{x}_{\ell}^{\prime}\right)}
\end{aligned}
$$


For all $F: X^{n} \times X^{n} \rightarrow \mathbb{C}$, we have

(a) $\left\|e^{-\varepsilon \mathrm{h}_{n}} F\right\|_{1, \infty} \leq e^{n \varepsilon\|\mathrm{h}\|_{1, \infty}}\|F\|_{1, \infty}$

(b) $\left\|e^{-\varepsilon V_{n}} F\right\|_{1, \infty} \leq e^{\frac{1}{2} n \varepsilon v_{0}}\|F\|_{1, \infty}$ where $v_{0}=\max _{\mathbf{x} \in X} v(\mathbf{x}, \mathbf{x})$

(c) $\left\|e^{-\varepsilon \mathrm{H}_{n}} F\right\|_{1, \infty} \leq e^{n \varepsilon\left(\|\mathrm{h}\|_{1, \infty}+\frac{1}{2} v_{0}\right)}\|F\|_{1, \infty}$

(d) $\left\|\mathrm{H}_{n} F\right\|_{1, \infty} \leq n\left(\|\mathrm{~h}\|_{1, \infty}+\frac{1}{2}(n-1)|v|_{X}\right)\|F\|_{1, \infty}$

Proof: (a) The kernel of $e^{-\varepsilon \mathrm{h}_{n}}$ is

$$
e^{-\varepsilon \mathrm{h}_{n}}\left(\tilde{\mathbf{x}}, \tilde{\mathbf{x}}^{\prime}\right)=\prod_{k=1}^{n} e^{-\varepsilon \mathrm{h}}\left(\mathbf{x}_{k}, \mathbf{x}_{k}^{\prime}\right)
$$

So we may view $e^{-\varepsilon \mathrm{h}_{n}} F$ as

$$
\overbrace{e^{-\varepsilon \mathrm{h}} \otimes \cdots \otimes e^{-\varepsilon \mathrm{h}}}^{n \text { factors }} \otimes \overbrace{\mathbb{1} \otimes \cdots \otimes \mathbb{1}}^{n \text { factors }}=\prod_{\prod_{k=1}}^{n} \overbrace{\mathbb{1} \otimes \cdots \otimes \mathbb{1}}^{k-1 \text { factors }} \otimes e^{-\varepsilon \mathrm{h}} \otimes \overbrace{\mathbb{1} \otimes \cdots \otimes \mathbb{1}}^{n-k \text { factors }}
$$

acting of $F$, viewed as an element of $L^{2}\left(X^{2 n}\right)$. The bounds

$$
\begin{aligned}
\sup _{\mathbf{x}} \int d \mathbf{x}^{\prime} \int d \mathbf{z}_{1} & \cdots d \mathbf{z}_{m}\left|L\left(\mathbf{x}, \mathbf{x}^{\prime}\right)\right|\left|G\left(\mathbf{x}^{\prime}, \mathbf{z}_{1}, \cdots, \mathbf{z}_{m}\right)\right| \\
& =\sup _{\mathbf{x}} \int d \mathbf{x}^{\prime}\left|L\left(\mathbf{x}, \mathbf{x}^{\prime}\right)\right| \int d \mathbf{z}_{1} \cdots d \mathbf{z}_{m}\left|G\left(\mathbf{x}^{\prime}, \mathbf{z}_{1}, \cdots, \mathbf{z}_{m}\right)\right| \\
& \leq\|L\|_{1, \infty}\|G\|_{1, \infty}
\end{aligned}
$$

and

$$
\begin{aligned}
\sup _{\mathbf{z}_{m}} \int d \mathbf{x} d \mathbf{x}^{\prime} \int & d \mathbf{z}_{1} \cdots d \mathbf{z}_{m-1}\left|L\left(\mathbf{x}, \mathbf{x}^{\prime}\right)\right|\left|G\left(\mathbf{x}^{\prime}, \mathbf{z}_{1}, \cdots, \mathbf{z}_{m}\right)\right| \\
& =\sup _{\mathbf{z}_{m}} \int d \mathbf{x}^{\prime} d \mathbf{z}_{1} \cdots d \mathbf{z}_{m-1}\left[\int d \mathbf{x}\left|L\left(\mathbf{x}, \mathbf{x}^{\prime}\right)\right|\right]\left|G\left(\mathbf{x}^{\prime}, \mathbf{z}_{1}, \cdots, \mathbf{z}_{m}\right)\right| \\
\leq & \|L\|_{1, \infty}\|G\|_{1, \infty}
\end{aligned}
$$

imply that

$$
\|\overbrace{\mathbb{1} \otimes \cdots \otimes \mathbb{1}}^{k-1 \text { factors }} \otimes L \otimes \overbrace{1 \otimes \cdots \otimes \mathbb{1}}^{n-k \text { factors }} F\|_{1, \infty} \leq\|L\|_{1, \infty}\|F\|_{1, \infty}
$$

Part (a) now follows by repeated application of (III.8) in conjunction with

$$
\begin{aligned}
\int d \mathbf{x}^{\prime}\left|e^{-\varepsilon \mathrm{h}}\left(\mathbf{x}^{\prime}, \mathbf{x}\right)\right| & =\int d \mathbf{x}^{\prime}\left|e^{-\varepsilon \mathrm{h}}\left(\mathbf{x}, \mathbf{x}^{\prime}\right)\right| \leq \sum_{n=0}^{\infty} \int d \mathbf{x}^{\prime} \frac{1}{n !} \varepsilon^{n}\left|\mathrm{~h}^{n}\left(\mathbf{x}, \mathbf{x}^{\prime}\right)\right| \\
& \leq \sum_{n=0}^{\infty} \frac{1}{n !} \varepsilon^{n}\|\mathrm{~h}\|_{1, \infty}^{n}=e^{\varepsilon\|\mathrm{h}\|_{1, \infty}}
\end{aligned}
$$


(b) Since $v(\mathbf{x}, \mathbf{y})$ is the kernel of a positive definite operator

$$
\sum_{\substack{j, k=1 \\ j \neq k}}^{n} v\left(\mathbf{x}_{j}, \mathbf{x}_{k}\right)=\sum_{j, k=1}^{n} v\left(\mathbf{x}_{j}, \mathbf{x}_{k}\right)-\sum_{j=1}^{n} v\left(\mathbf{x}_{j}, \mathbf{x}_{j}\right) \geq-v_{0} n
$$

so that

$$
\left|e^{-\varepsilon V_{n}} F(\tilde{\mathbf{x}}, \tilde{\mathbf{y}})\right| \leq e^{\frac{1}{2} n \varepsilon v_{0}}|F(\tilde{\mathbf{x}}, \tilde{\mathbf{y}})|
$$

(c) follows from the Trotter product formula

$$
e^{-\varepsilon \mathrm{H}_{n}} F=\lim _{p \rightarrow \infty}\left(e^{-\frac{\varepsilon}{p} \mathrm{~h}_{n}} e^{-\frac{\varepsilon}{p} V_{n}}\right)^{p} F
$$

and repeated application of parts (a) and (b).

(d) By (III.8),

$$
\left\|\mathrm{h}_{n} F\right\|_{1, \infty} \leq n\|\mathrm{~h}\|_{1, \infty}\|F\|_{1, \infty}
$$

Since

$$
\left|\sum_{\substack{j, k=1 \\ j \neq k}}^{n} v\left(\mathbf{x}_{j}, \mathbf{x}_{k}\right)\right| \leq n(n-1)|v|_{X}
$$

we also have

$$
\left\|V_{n} F\right\|_{1, \infty} \leq \frac{1}{2} n(n-1)|v|_{X}\|F\|_{1, \infty}
$$

and the claim follows.

Lemma III.11 The functions $F_{n}(\varepsilon, \tilde{\mathbf{x}}, \tilde{\mathbf{y}})$ of Lemma III.9 obey

$$
\left\|F_{n}(\varepsilon, \cdot, \cdot)\right\|_{1, \infty} \leq\left(8 \varepsilon\|v\|_{1, \infty}\right)^{n-1} e^{n \varepsilon K_{1}} \frac{1}{n^{3}}
$$

where $K_{1}=\|\mathrm{h}\|_{1, \infty}+\frac{v_{0}}{2}$. Furthermore there is a constant const, depending only on $\|\mathrm{h}\|_{1, \infty}$ and $\|v\|_{1, \infty}$, such that

$$
\begin{array}{r}
F_{2}\left(\varepsilon, \mathbf{x}_{1}, \mathbf{x}_{2}, \mathbf{y}_{1}, \mathbf{y}_{2}\right)=-\frac{1}{4} \varepsilon v\left(\mathbf{x}_{1}, \mathbf{x}_{2}\right)\left[\delta_{\mathbf{x}_{1}}\left(\mathbf{y}_{1}\right) \delta_{\mathbf{x}_{2}}\left(\mathbf{y}_{2}\right)+\delta_{\mathbf{x}_{1}}\left(\mathbf{y}_{2}\right) \delta_{\mathbf{x}_{2}}\left(\mathbf{y}_{1}\right)\right] \\
+ \\
+F_{2+}\left(\varepsilon, \mathbf{x}_{1}, \mathbf{x}_{2}, \mathbf{y}_{1}, \mathbf{y}_{2}\right)
\end{array}
$$

with

$$
\left\|F_{2+}(\varepsilon, \cdot, \cdot)\right\|_{1, \infty} \leq \operatorname{const} \varepsilon^{2}\|v\|_{1, \infty} e^{2 \varepsilon K_{1}}
$$


Proof: We first prove the bound on $\left\|F_{n}(\varepsilon, \cdot, \cdot)\right\|_{1, \infty}$ by induction on $n$. The case $n=1$ follows immediately from (III.9). So assume that the bound has been proven for all $m<n$. In general, if

$$
F\left(\mathbf{x}_{1}, \cdots, \mathbf{x}_{n+m}\right)=\int d \mathbf{y}_{1} d \mathbf{y}_{2} G\left(\mathbf{x}_{1}, \cdots, \mathbf{x}_{n}, \mathbf{y}_{1}\right) \omega\left(\mathbf{y}_{1}, \mathbf{y}_{2}\right) H\left(\mathbf{y}_{2}, \mathbf{x}_{n+1}, \cdots, \mathbf{x}_{n+m}\right)
$$

then

$$
\|F\|_{1, \infty} \leq\|G\|_{1, \infty}\|\omega\|_{1, \infty}\|H\|_{1, \infty}
$$

This is proven by repeated application of

$$
\begin{aligned}
\left|\int d \mathbf{x}_{2} d \mathbf{y} f\left(\mathbf{x}_{1}, \mathbf{y}\right) g\left(\mathbf{y}, \mathbf{x}_{2}\right)\right| & \leq \int d \mathbf{y}\left|f\left(\mathbf{x}_{1}, \mathbf{y}\right)\right| \int d \mathbf{x}_{2}\left|g\left(\mathbf{y}, \mathbf{x}_{2}\right)\right| \leq \int d \mathbf{y}\left|f\left(\mathbf{x}_{1}, \mathbf{y}\right)\right|\|g\|_{1, \infty} \\
& \leq\|f\|_{1, \infty}\|g\|_{1, \infty}
\end{aligned}
$$

Hence, by the inductive hypothesis, Lemma III.9 and part (c) of Lemma III.10,

$$
\begin{aligned}
\left\|F_{n}(\varepsilon, \tilde{\mathbf{x}}, \tilde{\mathbf{y}})\right\|_{1, \infty} & \leq \frac{1}{2} \| \int_{0}^{\varepsilon} d \tau \int d \tilde{\mathbf{x}}^{\prime}\left(e^{-(\varepsilon-\tau) \mathrm{H}_{n}}\right)\left(\tilde{\mathbf{x}}, \tilde{\mathbf{x}}^{\prime}\right) \sum_{m=1}^{n-1} S_{\tilde{\mathbf{x}}, \tilde{y}} \sum_{j=1}^{m} \sum_{k=m+1}^{n} v\left(\mathbf{x}_{j}^{\prime}, \mathbf{x}_{k}^{\prime}\right) \\
& F_{m}\left(\tau, \tilde{\mathbf{x}}_{[\leq m]}^{\prime}, \tilde{\mathbf{y}}_{[\leq m]}\right) F_{n-m}\left(\tau, \tilde{\mathbf{x}}_{[>m]}^{\prime}, \tilde{\mathbf{y}}_{[>m]}\right) \|_{1, \infty} \\
& \leq \frac{1}{2} \int_{0}^{\varepsilon} d \tau e^{(\varepsilon-\tau) K_{1} n} \sum_{m=1}^{n-1} \sum_{j=1}^{m} \sum_{k=m+1}^{n}\|v\|_{1, \infty}\left\|F_{m}(\tau, \cdot)\right\|_{1, \infty}\left\|F_{n-m}(\tau, \cdot)\right\|_{1, \infty} \\
& \leq \frac{1}{2} e^{n \varepsilon K_{1}}\left(8\|v\|_{1, \infty}\right)^{n-2} \int_{0}^{\varepsilon} d \tau \tau^{n-2} \sum_{m=1}^{n-1} \sum_{j=1}^{m} \sum_{k=m+1}^{n}\|v\|_{1, \infty} \frac{1}{m^{3}} \frac{1}{(n-m)^{3}} \\
& =\frac{1}{16} e^{n \varepsilon K_{1}}\left(8 \varepsilon\|v\|_{1, \infty}\right)^{n-1} \frac{1}{n-1} \sum_{m=1}^{n-1} \frac{1}{m^{2}} \frac{1}{(n-m)^{2}} \\
& \leq \frac{1}{16} e^{n \varepsilon K_{1}}\left(8 \varepsilon\|v\|_{1, \infty}\right)^{n-1} \frac{1}{n-1} \frac{8}{n^{2}} \\
& \leq\left(8 \varepsilon\|v\|_{1, \infty}\right)^{n-1} e^{n \varepsilon K_{1}} \frac{1}{n^{3}}
\end{aligned}
$$

The equation for $n=2$ in Lemma III.9 yields

$$
\begin{gathered}
F_{2}\left(\varepsilon, \mathbf{x}_{1}, \mathbf{x}_{2}, \mathbf{y}_{1}, \mathbf{y}_{2}\right)=-\frac{1}{2} \int_{0}^{\varepsilon} d \tau \int d \mathbf{x}_{1}^{\prime} d \mathbf{x}_{2}^{\prime}\left(e^{-(\varepsilon-\tau) \mathrm{H}_{2}}\right)\left(\mathbf{x}_{1}, \mathbf{x}_{2}, \mathbf{x}_{1}^{\prime}, \mathbf{x}_{2}^{\prime}\right) v\left(\mathbf{x}_{1}^{\prime}, \mathbf{x}_{2}^{\prime}\right) \\
\frac{1}{2}\left[e^{-\tau \mathrm{h}}\left(\mathbf{x}_{1}^{\prime}, \mathbf{y}_{1}\right) e^{-\tau \mathrm{h}}\left(\mathbf{x}_{2}^{\prime}, \mathbf{y}_{2}\right)+e^{-\tau \mathrm{h}}\left(\mathbf{x}_{1}^{\prime}, \mathbf{y}_{2}\right) e^{-\tau \mathrm{h}}\left(\mathbf{x}_{2}^{\prime}, \mathbf{y}_{1}\right)\right] \\
=-\frac{1}{4} \varepsilon v\left(\mathbf{x}_{1}, \mathbf{x}_{2}\right)\left[\delta_{\mathbf{x}_{1}}\left(\mathbf{y}_{1}\right) \delta_{\mathbf{x}_{2}}\left(\mathbf{y}_{2}\right)+\delta_{\mathbf{x}_{1}}\left(\mathbf{y}_{2}\right) \delta_{\mathbf{x}_{2}}\left(\mathbf{y}_{1}\right)\right] \\
+F_{2+}\left(\varepsilon, \mathbf{x}_{1}, \mathbf{x}_{2}, \mathbf{y}_{1}, \mathbf{y}_{2}\right)
\end{gathered}
$$

where the second order Taylor remainder

$$
F_{2+}\left(\varepsilon, \mathbf{x}_{1}, \mathbf{x}_{2}, \mathbf{y}_{1}, \mathbf{y}_{2}\right)=\int_{0}^{\varepsilon} d \tau^{\prime}\left(\varepsilon-\tau^{\prime}\right) \frac{\partial^{2}}{\partial \varepsilon^{2}} F_{2}\left(\tau^{\prime}, \mathbf{x}_{1}, \mathbf{x}_{2}, \mathbf{y}_{1}, \mathbf{y}_{2}\right)
$$


By (III.7)

$$
\begin{aligned}
\frac{\partial}{\partial \varepsilon} F_{2}\left(\varepsilon, \mathbf{x}_{1}, \mathbf{x}_{2}, \mathbf{y}_{1}, \mathbf{y}_{2}\right)=-\int & d \mathbf{x}_{1}^{\prime} d \mathbf{x}_{2}^{\prime} \mathrm{H}_{2}\left(\mathbf{x}_{1}, \mathbf{x}_{2}, \mathbf{x}_{1}^{\prime}, \mathbf{x}_{2}^{\prime}\right) F_{2}\left(\varepsilon, \mathbf{x}_{1}^{\prime}, \mathbf{x}_{2}^{\prime}, \mathbf{y}_{1}, \mathbf{y}_{2}\right) \\
& -\frac{1}{2} S_{\mathbf{x}, \mathbf{y}} v\left(\mathbf{x}_{1}, \mathbf{x}_{2}\right) F_{1}\left(\varepsilon, \mathbf{x}_{1}, \mathbf{y}_{1}\right) F_{1}\left(\varepsilon, \mathbf{x}_{2}, \mathbf{y}_{2}\right)
\end{aligned}
$$

and hence

$$
\begin{aligned}
\frac{\partial^{2}}{\partial \varepsilon^{2}} F_{2}\left(\varepsilon, \mathbf{x}_{1}, \mathbf{x}_{2}, \mathbf{y}_{1}, \mathbf{y}_{2}\right)=-\int & d \mathbf{x}_{1}^{\prime} d \mathbf{x}_{2}^{\prime} \mathrm{H}_{2}\left(\mathbf{x}_{1}, \mathbf{x}_{2}, \mathbf{x}_{1}^{\prime}, \mathbf{x}_{2}^{\prime}\right) \frac{\partial}{\partial \varepsilon} F_{2}\left(\varepsilon, \mathbf{x}_{1}^{\prime}, \mathbf{x}_{2}^{\prime}, \mathbf{y}_{1}, \mathbf{y}_{2}\right) \\
& -S_{\mathbf{x}, \mathbf{y}} v\left(\mathbf{x}_{1}, \mathbf{x}_{2}\right) F_{1}\left(\varepsilon, \mathbf{x}_{1}, \mathbf{y}_{1}\right) \frac{\partial}{\partial \varepsilon} F_{1}\left(\varepsilon, \mathbf{x}_{2}, \mathbf{y}_{2}\right)
\end{aligned}
$$

Using part (d) of Lemma III.10 and the bounds on $F_{1}$ and $F_{2}$ already proven, we have

$$
\begin{aligned}
\left\|\frac{\partial}{\partial \varepsilon} F_{2}(\varepsilon, \cdot, \cdot)\right\|_{1, \infty} & \leq\left\|\mathrm{H}_{2} F_{2}(\varepsilon, \cdot, \cdot)\right\|_{1, \infty}+\frac{1}{2}\|v\|_{1, \infty}\left\|F_{1}(\varepsilon, \cdot, \cdot)\right\|_{1, \infty}^{2} \\
& \leq 2\left(\|\mathrm{~h}\|_{1, \infty}+\frac{1}{2}|v|_{X}\right)\left\|F_{2}(\varepsilon, \cdot, \cdot)\right\|_{1, \infty}+\frac{1}{2}\|v\|_{1, \infty}\left\|F_{1}(\varepsilon, \cdot, \cdot)\right\|_{1, \infty}^{2} \\
& \leq \frac{1}{2}\|v\|_{1, \infty}\left[1+4 \varepsilon\left(\|\mathrm{h}\|_{1, \infty}+\frac{1}{2}|v|_{X}\right)\right] e^{2 \varepsilon K_{1}}
\end{aligned}
$$

Using the bound

$$
\left\|\frac{\partial}{\partial \varepsilon} F_{1}(\varepsilon, \cdot, \cdot)\right\|_{1, \infty}=\left\|\mathrm{h} e^{-\varepsilon \mathrm{h}}\right\|_{1, \infty} \leq\|\mathrm{h}\|_{1, \infty} e^{\varepsilon K_{1}}
$$

we similarly get

$$
\begin{aligned}
\| \frac{\partial^{2}}{\partial \varepsilon^{2}} F_{2} & (\varepsilon, \cdot, \cdot) \|_{1, \infty} \\
& \leq\left\|\mathrm{H}_{2} \frac{\partial}{\partial \varepsilon} F_{2}(\varepsilon, \cdot, \cdot)\right\|_{1, \infty}+\|v\|_{1, \infty}\left\|F_{1}(\varepsilon, \cdot, \cdot)\right\|_{1, \infty}\left\|\frac{\partial}{\partial \varepsilon} F_{1}(\varepsilon, \cdot, \cdot)\right\|_{1, \infty} \\
& \leq\left(\|\mathrm{h}\|_{1, \infty}+\frac{1}{2}|v|_{X}\right)\|v\|_{1, \infty}\left[1+4 \varepsilon\left(\|\mathrm{h}\|_{1, \infty}+\frac{1}{2}|v|_{X}\right)\right] e^{2 \varepsilon K_{1}}+\|v\|_{1, \infty}\|\mathrm{h}\|_{1, \infty} e^{2 \varepsilon K_{1}} \\
& \leq\left[\|v\|_{1, \infty}\left(2\|\mathrm{~h}\|_{1, \infty}+\frac{1}{2}|v|_{X}\right)+4 \varepsilon\|v\|_{1, \infty}\left(\|\mathrm{h}\|_{1, \infty}+\frac{1}{2}|v|_{X}\right)^{2}\right] e^{2 \varepsilon K_{1}} \\
& \leq \mathrm{const}\|v\|_{1, \infty} e^{2 \varepsilon K_{1}}
\end{aligned}
$$

Hence, by (III.11)

$$
\left\|F_{2+}(\varepsilon, \cdot, \cdot)\right\|_{1, \infty} \leq \operatorname{const} \varepsilon^{2}\|v\|_{1, \infty} e^{2 \varepsilon K_{1}}
$$

as desired.

Proof of Proposition III.6: We routinely write $\tilde{\mathbf{x}}$ for $\left(\mathbf{x}_{1}, \cdots, \mathbf{x}_{n}\right)$ and $\tilde{\mathbf{y}}$ for $\left(\mathbf{y}_{1}, \cdots, \mathbf{y}_{n}\right)$. The value of $n$ should be clear from the context. By Lemma III.11,

$$
\begin{gathered}
\sum_{n=3}^{\infty} \int d \tilde{\mathbf{x}} d \tilde{\mathbf{y}}\left|e^{n \varepsilon \mu} F_{n}(\varepsilon, \tilde{\mathbf{x}}, \tilde{\mathbf{y}}) \prod_{i=1}^{n} \alpha\left(\mathbf{x}_{i}\right)^{*} \phi\left(\mathbf{y}_{i}\right)\right| \leq \sum_{n=3}^{\infty}|X| e^{n \varepsilon \mu}\left\|F_{n}(\varepsilon, \cdot, \cdot)\right\|_{1, \infty}|\alpha|_{X}^{n}|\phi|_{X}^{n} \\
\leq|X| \sum_{n=3}^{\infty}\left(8 \varepsilon\|v\|_{1, \infty}\right)^{n-1} e^{n \varepsilon\left(K_{1}+\mu\right)}|\alpha|_{X}^{n}|\phi|_{X}^{n}
\end{gathered}
$$


This gives us the desired domain of analyticity. Since $\left\langle\alpha\left|e^{-\varepsilon K}\right| \phi\right\rangle$ and $e^{F\left(\varepsilon, \alpha^{*}, \phi\right)}$ are both analytic on this domain and since they agree for all sufficiently small $\alpha, \phi$, they coincide on the full domain.

Set

$$
\begin{array}{rl}
\mathcal{F}_{1}\left(\varepsilon, \alpha^{*}, \phi\right)=\int d \mathbf{x} & d \mathbf{y}\left[e^{\varepsilon \mu} F_{1}(\varepsilon, \mathbf{x}, \mathbf{y})-j(\varepsilon ; \mathbf{x}, \mathbf{y})\right] \alpha(\mathbf{x})^{*} \phi(\mathbf{y}) \\
& +\int d^{2} \tilde{\mathbf{x}} d^{2} \tilde{\mathbf{y}}\left[e^{2 \varepsilon \mu}-1\right] F_{2}(\varepsilon, \tilde{\mathbf{x}}, \tilde{\mathbf{y}}) \prod_{i=1}^{2} \alpha\left(\mathbf{x}_{i}\right)^{*} \phi\left(\mathbf{y}_{i}\right) \\
& +\int d^{2} \tilde{\mathbf{x}} d^{2} \tilde{\mathbf{y}} F_{2+}(\varepsilon, \tilde{\mathbf{x}}, \tilde{\mathbf{y}}) \prod_{i=1}^{2} \alpha\left(\mathbf{x}_{i}\right)^{*} \phi\left(\mathbf{y}_{i}\right) \\
& +\sum_{n=3}^{\infty} \int d^{n} \tilde{\mathbf{x}} d^{n} \tilde{\mathbf{y}} e^{n \varepsilon \mu} F_{n}(\varepsilon, \tilde{\mathbf{x}}, \tilde{\mathbf{y}}) \prod_{i=1}^{n} \alpha\left(\mathbf{x}_{i}\right)^{*} \phi\left(\mathbf{y}_{i}\right)
\end{array}
$$

so that

$$
\begin{aligned}
F\left(\varepsilon, \alpha^{*}, \phi\right)=\iint_{X^{2}} d \mathbf{x} d \mathbf{y} \alpha & (\mathbf{x})^{*} j(\varepsilon ; \mathbf{x}, \mathbf{y}) \phi(\mathbf{y}) \\
& -\frac{\varepsilon}{2} \iint_{X^{2}} d \mathbf{x} d \mathbf{y} \alpha(\mathbf{x})^{*} \alpha(\mathbf{y})^{*} v(\mathbf{x}, \mathbf{y}) \phi(\mathbf{x}) \phi(\mathbf{y})+\mathcal{F}_{1}\left(\varepsilon, \alpha^{*}, \phi\right)
\end{aligned}
$$

If $|\alpha|_{X},|\phi|_{X} \leq R$, then, by (III.12),

$$
\begin{aligned}
\left|\sum_{n=3}^{\infty} \int d^{n} \tilde{\mathbf{x}} d^{n} \tilde{\mathbf{y}} e^{n \varepsilon \mu} F_{n}(\varepsilon, \tilde{\mathbf{x}}, \tilde{\mathbf{y}}) \prod_{i=1}^{n} \alpha\left(\mathbf{x}_{i}\right)^{*} \phi\left(\mathbf{y}_{i}\right)\right| & \leq|X| \sum_{n=3}^{\infty}\left(8 \varepsilon\|v\|_{1, \infty}\right)^{n-1} e^{n \varepsilon\left(K_{1}+\mu\right)} R^{2 n} \\
& \leq \frac{64|X| \varepsilon^{2}\|v\|_{1, \infty}^{2} e^{3 \varepsilon\left(K_{1}+\mu\right)} R^{6}}{1-8 \varepsilon\|v\|_{1, \infty} e^{\varepsilon\left(K_{1}+\mu\right)} R^{2}} \\
& \leq 128 \varepsilon^{2} R^{6}|X|\|v\|_{1, \infty}^{2} e^{3 \varepsilon\left(K_{1}+\mu\right)}
\end{aligned}
$$

Similarly, by Lemma III.11,

$$
\begin{aligned}
\left|\int d^{2} \tilde{\mathbf{x}} d^{2} \tilde{\mathbf{y}} F_{2+}(\varepsilon, \tilde{\mathbf{x}}, \tilde{\mathbf{y}}) \prod_{i=1}^{2} \alpha\left(\mathbf{x}_{i}\right)^{*} \phi\left(\mathbf{y}_{i}\right)\right| & \leq|X|\left\|F_{2+}(\varepsilon, \cdot, \cdot)\right\|_{1, \infty} R^{4} \\
& \leq \operatorname{const} \varepsilon^{2} R^{4}|X|\|v\|_{1, \infty} e^{2 \varepsilon\left(K_{1}+|\mu|\right)}
\end{aligned}
$$

and

$$
\begin{aligned}
\left|\int d^{2} \tilde{\mathbf{x}} d^{2} \tilde{\mathbf{y}}\left[e^{2 \varepsilon \mu}-1\right] F_{2}(\varepsilon, \tilde{\mathbf{x}}, \tilde{\mathbf{y}}) \prod_{i=1}^{2} \alpha\left(\mathbf{x}_{i}\right)^{*} \phi\left(\mathbf{y}_{i}\right)\right| & \leq 2 \varepsilon|\mu||X|\left\|F_{2}(\varepsilon, \cdot, \cdot)\right\|_{1, \infty} R^{4} e^{2 \varepsilon|\mu|} \\
& \leq 2|\mu| \varepsilon^{2} R^{4}|X|\|v\|_{1, \infty} e^{2 \varepsilon\left(K_{1}+|\mu|\right)}
\end{aligned}
$$

and

$$
\begin{aligned}
\left|\int d \mathbf{x} d \mathbf{y}\left[e^{\varepsilon \mu} F_{1}(\varepsilon, \mathbf{x}, \mathbf{y})-j(\varepsilon ; \mathbf{x}, \mathbf{y})\right] \alpha(\mathbf{x})^{*} \phi(\mathbf{y})\right| \\
\quad \leq|X|\left\|e^{-\varepsilon(\mathrm{h}-\mu)}-j(\varepsilon)\right\|_{1, \infty} R^{2} \\
\leq c_{j} \varepsilon^{2} R^{2}|X|
\end{aligned}
$$


Since

$$
R^{4}\|v\|_{1, \infty}=R\left(R^{3}\|v\|_{1, \infty}\right) \leq \frac{1}{2}\left(R^{2}+\|v\|_{1, \infty}^{2} R^{6}\right)
$$

the desired bound on $\mathcal{F}_{1}$ follows.

Example III.12 Here is a simple example that shows that $\left\langle\alpha\left|e^{-\varepsilon K}\right| \phi\right\rangle$ can have zeroes so that the logarithm of $\left\langle\alpha\left|e^{-\varepsilon K}\right| \phi\right\rangle$ need not be defined for all $\alpha, \phi \in L^{2}(X)$. If the finite set $X$, which plays the role of space here, consists of just a single point, then each $n$-particle space $\mathcal{B}_{n}$ with $n \geq 1$ has dimension exactly one. So any operator that commutes with the number operator must be a function of the number operator. In particular, $K=H_{0}+V-\mu N$, which is of degree two in the annihilation operators and of degree two in the creation operators, is a polynomial in $N$ of degree two. As a simple example, we take $K=N^{2}-N$. Then

$$
\left\langle\alpha\left|e^{-\varepsilon\left(N^{2}-N\right)}\right| \phi\right\rangle=\sum_{n=0}^{\infty} \frac{\left(\alpha^{*} \phi\right)^{n}}{n !} e^{-\varepsilon n(n-1)}
$$

Set

$$
f(z)=\sum_{n=0}^{\infty} \frac{z^{n}}{n !} e^{-\varepsilon n(n-1)}
$$

Observe that $f$ fulfills the functional equation

$$
f^{\prime}(z)=f\left(e^{-2 \varepsilon} z\right)
$$

since

$$
\begin{aligned}
f\left(e^{-2 \varepsilon} z\right) & =\sum_{n=0}^{\infty} \frac{z^{n}}{n !} e^{-2 \varepsilon n} e^{-\varepsilon n(n-1)}=\sum_{n=0}^{\infty} \frac{z^{n}}{n !} e^{-\varepsilon n(n+1)}=\frac{d}{d z} \sum_{n=0}^{\infty} \frac{z^{n+1}}{(n+1) !} e^{-\varepsilon n(n+1)} \\
= & \frac{d}{d z} \sum_{n=1}^{\infty} \frac{z^{n}}{n !} e^{-\varepsilon n(n-1)}=\frac{d}{d z} f(z)
\end{aligned}
$$

We claim that $f$ necessarily has a zero on the negative real axis, somewhere between 0 and $-\frac{1}{1-e^{-2 \varepsilon}}=-\frac{1}{2 \varepsilon+O\left(\varepsilon^{2}\right)}$.

Proof: Set $\kappa=e^{-2 \varepsilon}<1$, so that $f^{\prime}(z)=f(\kappa z)$. By inspection, $f(x)>0$ for all $x \geq 0$. Now assume that $f$ has no zero on the negative real axis. Then

$$
h(x)=-\log f(-x)
$$

is well defined for all real $x$ and fulfills the equation

$$
\begin{aligned}
h^{\prime}(x) & =\frac{f^{\prime}(-x)}{f(-x)}=\frac{f(-\kappa x)}{f(-x)} \\
& =e^{h(x)-h(\kappa x)}
\end{aligned}
$$


In particular $h^{\prime}(x)>0$ for all $x \in \mathbb{R}$ and $h$ is monotonically increasing. The second derivative

$$
\begin{aligned}
h^{\prime \prime}(x) & =\left(h^{\prime}(x)-\kappa h^{\prime}(\kappa x)\right) e^{h(x)-h(\kappa x)} \\
& =\left(e^{h(x)-h(\kappa x)}-\kappa e^{h(\kappa x)-h\left(\kappa^{2} x\right)}\right) e^{h(x)-h(\kappa x)}
\end{aligned}
$$

As $\kappa<1$ and $h(0)=0$, we have $h^{\prime \prime}(0)=1-\kappa>0$. Next we show that $h^{\prime \prime}(x)>0$ for all $x \geq 0$. If this were not the case we would have a smallest positive zero $x_{0}$ of $h^{\prime \prime}$. Then $h^{\prime}$ would be monotonically increasing in $\left[0, x_{0}\right]$. By the formula for $h^{\prime \prime}$ above

$$
e^{h\left(x_{0}\right)-h\left(\kappa x_{0}\right)}-\kappa e^{h\left(\kappa x_{0}\right)-h\left(\kappa^{2} x_{0}\right)}=0
$$

so that

$$
h\left(x_{0}\right)-h\left(\kappa x_{0}\right)<h\left(\kappa x_{0}\right)-h\left(\kappa^{2} x_{0}\right)
$$

By the mean value theorem there exist $\xi_{1} \in\left[\kappa x_{0}, x_{0}\right]$ and $\xi_{2} \in\left[\kappa^{2} x_{0}, \kappa x_{0}\right]$ such that

$$
\begin{aligned}
h\left(x_{0}\right)-h\left(\kappa x_{0}\right) & =h^{\prime}\left(\xi_{1}\right)(1-\kappa) x_{0} \\
h\left(\kappa x_{0}\right)-h\left(\kappa^{2} x_{0}\right) & =h^{\prime}\left(\xi_{2}\right)(1-\kappa) \kappa x_{0}
\end{aligned}
$$

Then

$$
h^{\prime}\left(\xi_{1}\right)(1-\kappa) x_{0}<h^{\prime}\left(\xi_{2}\right)(1-\kappa) \kappa x_{0}
$$

and

$$
h^{\prime}\left(\xi_{1}\right)<h^{\prime}\left(\xi_{2}\right) \kappa
$$

As $\xi_{2} \leq \xi_{1} \leq x_{0}$ and $\kappa<1$ this contradicts the monotonicity of $h^{\prime}$ on $\left[0, x_{0}\right]$.

Since $h(0)=0$ and $h^{\prime \prime}(x)>0$ for all $x \geq 0$ we have $h(\kappa x) \leq \kappa h(x)$ for all $x \geq 0$ and therefore $h(x)-h(\kappa x) \geq(1-\kappa) h(x)$. In particular

$$
h^{\prime}(x)=e^{h(x)-h(\kappa x)} \geq e^{(1-\kappa) h(x)} \Rightarrow e^{-(1-\kappa) h(x)} h^{\prime}(x) \geq 1 \Rightarrow-\frac{1}{1-\kappa} \frac{d}{d x} e^{-(1-\kappa) h(x)} \geq 1
$$

Integrating both sides and using the initial condition $h(0)=0$ gives

$$
-\frac{1}{1-\kappa}\left[e^{-(1-\kappa) h(x)}-1\right] \geq x \Rightarrow e^{-(1-\kappa) h(x)} \leq 1-(1-\kappa) x
$$

for all $x \geq 0$. Thus $h(x)$ must have a pole at some $0<x<\frac{1}{1-\kappa}$ and $f(x)$ must have a zero at some $-\frac{1}{1-\kappa}<x<0$. 


\section{A Functional Integral Representation of $\operatorname{Tr} e^{-\beta K}$}

Let $\mathrm{h}$ be a single particle operator on $X$ and $v\left(\mathbf{x}_{1}, \mathbf{x}_{2}\right)$ a real, symmetric, pair potential which is repulsive in the sense of Definition II.16. One of our precise formulations of the standard physics representation (I.1) for the partition function $\operatorname{Tr} e^{-\beta K}$, where $K=$ $\mathrm{H}_{0}(\mathrm{~h}, X)+\mathrm{V}(v, X)-\mu N$ is the second quantized Hamiltonian of a boson gas, is

Theorem III.13 Suppose that the sequence $\mathrm{R}(p)$ obeys

$$
\lim _{p \rightarrow \infty} p e^{-\frac{1}{2} \mathrm{R}(p)^{2}}=0 \quad \text { and } \quad \mathrm{R}(p)<p^{\frac{1}{24|X|}}
$$

Then

$$
\operatorname{Tr} e^{-\beta K}=\lim _{p \rightarrow \infty} \int \prod_{\tau \in \mathcal{T}_{p}}\left[d \mu_{\mathrm{R}(p)}\left(\phi_{\tau}^{*}, \phi_{\tau}\right) e^{-\int d \mathbf{y}\left[\phi_{\tau}^{*}(\mathbf{y})-\phi_{\tau-\varepsilon}^{*}(\mathbf{y})\right] \phi_{\tau}(\mathbf{y})} e^{-\varepsilon K\left(\phi_{\tau-\varepsilon}^{*}, \phi_{\tau}\right)}\right]
$$

with the conventions that $\varepsilon=\frac{\beta}{p}$ and $\phi_{0}=\phi_{p \varepsilon}$. The function $K\left(\alpha^{*}, \phi\right)$ was defined in Corollary III. \%.

This Theorem will be proven after Example III.17. During the course of the proof, we will modify the factors $\left\langle\phi_{\tau-\varepsilon}\left|e^{-\varepsilon K}\right| \phi_{\tau}\right\rangle$ of the integrand in Theorem III.1, using the representation of these factors in Corollary III.7. In Proposition III.16, below, we develop some machinery to assist in proving that such modifications do not change the limit.

Definition III.14 Let $r>0$. Define, for $\mathcal{I}: \mathbb{C}^{2|X|} \rightarrow \mathbb{C}$, the seminorm

$$
\|\mathcal{I}\|_{r}=\sup _{\substack{\alpha, \phi \in \mathbb{G}^{X} \\|\alpha| X,|\phi|_{X} \leq r}}|\mathcal{I}(\alpha, \phi)|
$$

The "r-product" of $\mathcal{I}, \mathcal{J}: \mathbb{C}^{2|X|} \rightarrow \mathbb{C}$, with $\|\mathcal{I}\|_{r},\|\mathcal{J}\|_{r}<\infty$ is defined to be

$$
\left(\mathcal{I} *_{r} \mathcal{J}\right)(\alpha, \gamma)=\int \mathcal{I}(\alpha, \phi) \mathcal{J}(\phi, \gamma) d \mu_{r}\left(\phi^{*}, \phi\right)
$$

which is just the convolution with respect to the measure $d \mu_{r}$. The $q^{\text {th }}$ power with respect to this product is denoted

$$
\mathcal{I}^{*_{r} q}=\overbrace{\mathcal{I} *_{r} \mathcal{I} *_{r} \cdots *_{r} \mathcal{I}}^{q \text { factors }}
$$

Example III.15 For each $\varepsilon>0$, set

$$
\mathcal{I}_{\varepsilon}(\alpha, \phi)=e^{-\frac{1}{2}\|\alpha\|^{2}-\frac{1}{2}\|\phi\|^{2}} e^{F\left(\varepsilon, \alpha^{*}, \phi\right)}=e^{-\frac{1}{2}\|\alpha\|^{2}-\frac{1}{2}\|\phi\|^{2}}\left\langle\alpha\left|e^{-\varepsilon K}\right| \phi\right\rangle
$$


Theorem III.1 states that, for $\mathrm{R}(p)$ obeying $\lim _{p \rightarrow \infty} p e^{-\frac{1}{2} \mathrm{R}(p)^{2}}=0$,

$$
\operatorname{Tr} e^{-\beta K}=\left.\lim _{p \rightarrow \infty} \int d \mu_{r}\left(\phi^{*}, \phi\right) \mathcal{I}_{\varepsilon}^{*_{r} p}(\phi, \phi)\right|_{\substack{r=\mathrm{R}(p) \\ \varepsilon=\beta / p}}
$$

The operator $K$ is bounded below. Suppose that $K \geq-K_{0} 1$. Then

$$
\left|\left\langle\alpha\left|e^{-\varepsilon K}\right| \phi\right\rangle\right| \leq\|\alpha\| e^{\varepsilon K_{0}}\|\phi\|=e^{\frac{1}{2}\|\alpha\|^{2}+\frac{1}{2}\|\phi\|^{2}} e^{\varepsilon K_{0}}
$$

implies that

$$
\left\|\mathcal{I}_{\varepsilon}\right\|_{r} \leq e^{\varepsilon K_{0}}
$$

for all $r>0$. Furthermore

$$
\mathcal{I}_{\varepsilon}^{*_{r} q}(\alpha, \phi)=e^{-\frac{1}{2}\|\alpha\|^{2}-\frac{1}{2}\|\phi\|^{2}}\left\langle\alpha\left|\left(e^{-\varepsilon K} \mathrm{I}_{r}\right)^{q-1} e^{-\varepsilon K}\right| \phi\right\rangle
$$

Thus, by part (c) of Theorem II.26,

$$
\left\|\mathcal{I}_{\varepsilon}^{*_{r} q}\right\|_{r} \leq e^{q \varepsilon K_{0}}
$$

for all $r>0$.

Proposition III.16 Let $K_{0}, \varepsilon, \zeta>0$ and $0<\kappa<1$ and $r, C_{\beta} \geq 1$ obey

$$
C_{\beta}\left(\pi r^{2}\right)^{3|X|} \zeta^{1-\kappa} \leq \varepsilon
$$

Let $\mathcal{I}, \tilde{\mathcal{I}}: \mathbb{C}^{2|X|} \rightarrow \mathbb{C}$ obey

$$
\|\mathcal{I}-\tilde{\mathcal{I}}\|_{r} \leq \zeta \quad\left\|\mathcal{I}^{*_{r} q}\right\|_{r} \leq e^{q \varepsilon K_{0}} \text { for all } q \in \mathbb{N}
$$

Then, for all $q \in \mathbb{N}$ with $q \leq \frac{C_{\beta}}{\varepsilon}$,

$$
\begin{aligned}
\left\|\tilde{\mathcal{I}}^{*_{r} q}\right\|_{r} & \leq e^{q \varepsilon\left(K_{0}+\zeta^{\kappa}\right)} \\
\left\|\tilde{\mathcal{I}}^{*_{r} q}-\mathcal{I}^{*_{r} q}\right\|_{r} & \leq \zeta^{\kappa} e^{q \varepsilon\left(K_{0}+\zeta^{\kappa}\right)} \\
\int d \mu_{r}\left(\phi^{*}, \phi\right)\left|\tilde{\mathcal{I}}^{*_{r}} q(\phi, \phi)-\mathcal{I}^{*_{r}} q(\phi, \phi)\right| & \leq \zeta^{\kappa} e^{q \varepsilon\left(K_{0}+\zeta^{\kappa}\right)}
\end{aligned}
$$

Proof: For notational convenience, we suppress the subscript $r$ on $*_{r}$. We first prove, by induction on $q$, that

$$
\left\|\tilde{\mathcal{I}}^{* q}\right\|_{r} \leq(A+B)^{q} \quad\left\|\tilde{\mathcal{I}}^{* q}-\mathcal{I}^{* q}\right\|_{r} \leq q B(A+B)^{q-1}
$$


where $A=e^{\varepsilon K_{0}}$ and $B=\left(\pi r^{2}\right)^{2|X|} \zeta$. The case $q=1$ is obvious. So assume that these bounds hold when $q$ is replaced by $\ell<q$. Then

$$
\tilde{\mathcal{I}}^{* q}=\sum_{\ell=0}^{q-1} \mathcal{I}^{* \ell} *(\tilde{\mathcal{I}}-\mathcal{I}) * \tilde{\mathcal{I}}^{* q-\ell-1}+\mathcal{I}^{* q}
$$

Since

$$
\int d \mu_{r}\left(\phi^{*}, \phi\right) \leq\left(\pi r^{2}\right)^{|X|}
$$

we have

$$
\begin{aligned}
\left\|\tilde{\mathcal{I}}^{* q}\right\|_{r} & \leq \sum_{\ell=0}^{q-1}\left\|\mathcal{I}^{* \ell}\right\|_{r}\left(\pi r^{2}\right)^{|X|}\|\tilde{\mathcal{I}}-\mathcal{I}\|_{r}\left(\pi r^{2}\right)^{|X|}\left\|\tilde{\mathcal{I}}^{* q-\ell-1}\right\|_{r}+\left\|\mathcal{I}^{* q}\right\|_{r} \\
& \leq \sum_{\ell=0}^{q-1} A^{\ell} B(A+B)^{q-\ell-1}+A^{q}
\end{aligned}
$$

and

$$
\left\|\tilde{\mathcal{I}}^{* q}-\mathcal{I}^{* q}\right\|_{r} \leq \sum_{\ell=0}^{q-1} A^{\ell} B(A+B)^{q-\ell-1}
$$

Then (III.13) follows from

$$
\begin{aligned}
(A+B)^{q} & =\sum_{\ell=0}^{q-1} A^{\ell}(A+B)^{q-\ell}-\sum_{\ell=0}^{q-1} A^{\ell+1}(A+B)^{q-\ell-1}+A^{q} \\
& =\sum_{\ell=0}^{q-1} A^{\ell} B(A+B)^{q-\ell-1}+A^{q}
\end{aligned}
$$

and

$$
(A+B)^{q}-A^{q}=\int_{0}^{1} d t \frac{d}{d t}(A+t B)^{q} \leq q B(A+B)^{q-1}
$$

To complete the proof, we observe that

$$
\begin{aligned}
A+B & =e^{\varepsilon K_{0}}+\left(\pi r^{2}\right)^{2|X|} \zeta \leq e^{\varepsilon K_{0}}\left(1+\left(\pi r^{2}\right)^{2|X|} \zeta\right) \leq e^{\varepsilon K_{0}}\left(1+\varepsilon \zeta^{\kappa}\right) \\
& \leq e^{\varepsilon\left(K_{0}+\zeta^{\kappa}\right)}
\end{aligned}
$$

and

$$
q B, q B\left(\pi r^{2}\right)^{|X|} \leq \frac{C_{\beta}}{\varepsilon}\left(\pi r^{2}\right)^{3|X|} \zeta \leq \zeta^{\kappa}
$$




\section{Example III.17 Let}

$$
\mathcal{I}_{\varepsilon}(\alpha, \phi)=e^{-\frac{1}{2}\|\alpha\|^{2}-\frac{1}{2}\|\phi\|^{2}} e^{F\left(\varepsilon, \alpha^{*}, \phi\right)}=e^{-\frac{1}{2}\|\alpha\|^{2}-\frac{1}{2}\|\phi\|^{2}}\left\langle\alpha\left|e^{-\varepsilon K}\right| \phi\right\rangle
$$

be as in Example III.15 and set

$$
\tilde{\mathcal{I}}_{\varepsilon}(\alpha, \phi)=e^{-\frac{1}{2}\|\alpha\|^{2}-\frac{1}{2}\|\phi\|^{2}} e^{F\left(\varepsilon, \alpha^{*}, \phi\right)-\mathcal{F}_{0}\left(\varepsilon, \alpha^{*}, \phi\right)}=e^{-\frac{1}{2}\|\alpha\|^{2}-\frac{1}{2}\|\phi\|^{2}}\left\langle\alpha\left|e^{-\varepsilon K}\right| \phi\right\rangle e^{-\mathcal{F}_{0}\left(\varepsilon, \alpha^{*}, \phi\right)}
$$

where $\mathcal{F}_{0}$ was defined in Corollary III.7. Observe that

$$
\tilde{\mathcal{I}}_{\varepsilon}(\alpha, \phi)=\exp \left\{-\frac{1}{2}\|\alpha\|^{2}-\frac{1}{2}\|\phi\|^{2}+\int d \mathbf{x} \alpha^{*}(\mathbf{x}) \phi(\mathbf{x})-\varepsilon K\left(\alpha^{*}, \phi\right)\right\}
$$

Let $r$ satisfy $r \leq \frac{1}{2}\left[8 e^{\varepsilon\left(\|\mathrm{h}\|_{1, \infty}+\mu+v_{0}\right)} \varepsilon\|v\|_{1, \infty}\right]^{-1 / 2}$. Then, by Corollary III.7,

$$
\left|\mathcal{F}_{0}\left(\varepsilon, \alpha^{*}, \phi\right)\right| \leq \operatorname{const} \varepsilon^{2}\left(r^{2}+\|v\|_{1, \infty}^{2} r^{6}\right)|X| \quad \text { for all }|\alpha|_{X},|\phi|_{X} \leq r
$$

Consequently (assuming that $r>1$ and allowing the constant to depend on $\|v\|_{1, \infty}$ too)

$$
\begin{aligned}
\left\|\mathcal{I}_{\varepsilon}-\tilde{\mathcal{I}}_{\varepsilon}\right\|_{r} & =\sup _{|\alpha|_{X},|\phi|_{X} \leq r}\left|e^{-\frac{1}{2}\|\alpha\|^{2}-\frac{1}{2}\|\phi\|^{2}}\left\langle\alpha\left|e^{-\varepsilon K}\right| \phi\right\rangle\left[1-e^{-\mathcal{F}_{0}\left(\varepsilon, \alpha^{*}, \phi\right)}\right]\right| \\
& \leq e^{\varepsilon K_{0}} \text { const } \varepsilon^{2} r^{6}|X| e^{\text {const } \varepsilon^{2} r^{6}|X|}
\end{aligned}
$$

Proof of Theorem III.13: We apply Proposition III.16 with $\mathcal{I}=\mathcal{I}_{\varepsilon}, \tilde{\mathcal{I}}=\tilde{\mathcal{I}}_{\varepsilon}$, as in Examples III.15 and III.17, $\zeta=\varepsilon^{3 / 2}, r=\mathrm{R}(p), p=\frac{\beta}{\varepsilon}, \kappa=\frac{1}{12}$ and $C_{\beta}=\beta$. If $\varepsilon$ is sufficiently small, the three hypotheses of the Proposition are satisfied because then

$$
C_{\beta}\left(\pi r^{2}\right)^{3|X|} \zeta^{1-\kappa}=\beta \pi^{3|X|} \mathrm{R}(p)^{6|X|} \varepsilon^{\frac{3}{2}\left(1-\frac{1}{12}\right)} \leq \beta \pi^{3|X|}\left(\frac{\beta}{\varepsilon}\right)^{\frac{6}{24}} \varepsilon^{\frac{33}{24}} \leq \varepsilon
$$

and, by Example III.17,

$$
\|\mathcal{I}-\tilde{\mathcal{I}}\|_{r} \leq \operatorname{const} \varepsilon^{2}\left(\frac{\beta}{\varepsilon}\right)^{\frac{1}{4|X|}}|X| e^{\varepsilon K_{0}} e^{\text {const }|X| \varepsilon^{2}(\beta / \varepsilon)^{\frac{1}{4|X|}}} \leq \varepsilon^{3 / 2}=\zeta
$$

and, by Example III.15,

$$
\left\|\mathcal{I}^{*_{r} q}\right\|_{r} \leq e^{q \varepsilon K_{0}}
$$

By the last conclusion of Proposition III.16,

$$
\lim _{p \rightarrow \infty} \int d \mu_{r}\left(\alpha^{*}, \alpha\right)\left|\tilde{\mathcal{I}}_{\varepsilon}^{*_{r} p}(\alpha, \alpha)-\mathcal{I}_{\varepsilon}^{*_{r} p}(\alpha, \alpha)\right|_{\substack{r=\mathrm{R}(p) \\ \varepsilon=\beta / p}}=0
$$

Recall, from Example III.15, that

$$
\operatorname{Tr} e^{-\beta K}=\left.\lim _{p \rightarrow \infty} \int d \mu_{r}\left(\phi^{*}, \phi\right) \mathcal{I}_{\varepsilon}^{*_{r} p}(\phi, \phi)\right|_{\substack{r=\mathrm{R}(p) \\ \varepsilon=\beta / p}}
$$


so that

$$
\begin{aligned}
\operatorname{Tr} e^{-\beta K} & =\left.\lim _{p \rightarrow \infty} \int d \mu_{r}\left(\phi^{*}, \phi\right) \tilde{\mathcal{I}}_{\varepsilon}^{*} p(\phi, \phi)\right|_{\substack{r=\mathrm{R}(p) \\
\varepsilon=\beta / p}} \\
& =\lim _{p \rightarrow \infty} \int \prod_{\tau \in \mathcal{T}_{p}}\left[d \mu_{\mathrm{R}(p)}\left(\phi_{\tau}^{*}, \phi_{\tau}\right) e^{-\frac{1}{2}\left\|\phi_{\tau-\varepsilon}\right\|^{2}-\frac{1}{2}\left\|\phi_{\tau}\right\|^{2}+\int d \mathbf{y} \phi_{\tau-\varepsilon}^{*}(\mathbf{y}) \phi_{\tau}(\mathbf{y})} e^{-\varepsilon K\left(\phi_{\tau-\varepsilon}^{*}, \phi_{\tau}\right)}\right] \\
& =\lim _{p \rightarrow \infty} \int \prod_{\tau \in \mathcal{T}_{p}}\left[d \mu_{\mathrm{R}(p)}\left(\phi_{\tau}^{*}, \phi_{\tau}\right) e^{-\left\|\phi_{\tau}\right\|^{2}+\int d \mathbf{y} \phi_{\tau-\varepsilon}^{*}(\mathbf{y}) \phi_{\tau}(\mathbf{y})} e^{-\varepsilon K\left(\phi_{\tau-\varepsilon}^{*}, \phi_{\tau}\right)}\right] \\
& =\lim _{p \rightarrow \infty} \int \prod_{\tau \in \mathcal{T}_{p}}\left[d \mu_{\mathrm{R}(p)}\left(\phi_{\tau}^{*}, \phi_{\tau}\right) e^{-\int d \mathbf{y}\left[\phi_{\tau}^{*}(\mathbf{y})-\phi_{\tau-\varepsilon}^{*}(\mathbf{y})\right] \phi_{\tau}(\mathbf{y})} e^{-\varepsilon K\left(\phi_{\tau-\varepsilon}^{*}, \phi_{\tau}\right)}\right]
\end{aligned}
$$




\section{Appendix A: A Cameron Style Model Computation}

We consider the formal infinite dimensional complex Gaussian measure

$$
\frac{1}{N} \prod_{0 \leq \tau<1} \frac{d \phi_{\tau}^{*} d \phi_{\tau}}{2 \pi i} e^{\int_{0}^{1} d \tau \phi_{\tau}^{*}(\partial+\mu) \phi_{\tau}}
$$

where $\mu<0$,

$$
\partial \phi_{\tau}=\lim _{\varepsilon \rightarrow 0+} \frac{1}{\varepsilon}\left[\phi_{\tau+\varepsilon}-\phi_{\tau}\right]
$$

and the normalization constant $N$ is chosen so that the integral of the function 1 with respect to this measure is one. This is the formal Gaussian measure of (I.1) when $\beta=1$, $\mathbb{R}^{3}$ is replace by a single point and $\mathrm{h}=v=0$. Suppose one attempts to construct this measure as the limit

$$
\lim _{p \rightarrow \infty} \frac{1}{N_{p}} \prod_{\tau \in \mathrm{T}_{p}} \frac{d \phi_{\tau}^{*} d \phi_{\tau}}{2 \pi i} \quad \exp \left\{\frac{1}{p} \sum_{\tau \in \mathrm{T}_{p}}\left[\phi_{\tau}^{*} p\left(\phi_{\tau+1 / p}-\phi_{\tau}\right)+\mu \phi_{\tau}^{*} \phi_{\tau}\right]\right\}
$$

of finite dimensional complex Gaussian measures. Here, $\mathrm{T}_{p}=\left\{\frac{q}{p} \mid q=0, \cdots, p-1\right\}$ and we use the convention that $\phi_{1}=\phi_{0}$. The normalization constant

$$
N_{p}=\int d \nu_{p}\left(\phi^{*}, \phi\right)
$$

where

$$
d \nu_{p}\left(\phi^{*}, \phi\right)=\prod_{\tau \in \mathrm{T}_{p}} \frac{d \phi_{\tau}^{*} d \phi_{\tau}}{2 \pi i} \exp \left\{\frac{1}{p} \sum_{\tau \in \mathrm{T}_{p}}\left[\phi_{\tau}^{*} p\left(\phi_{\tau+1 / p}-\phi_{\tau}\right)+\mu \phi_{\tau}^{*} \phi_{\tau}\right]\right\}
$$

The following proposition shows that (A.1) cannot be a well-defined complex measure.

Proposition A.1 Let $\mu<0$. Then

$$
\lim _{p \rightarrow \infty} N_{p}=\frac{1}{e^{-\mu}-1}
$$

However, if $p$ is a multiple of 8 and is large enough, then

$$
\int\left|d \nu_{p}\left(\phi^{*}, \phi\right)\right|>10^{p / 8}
$$

Proof: Think of $\mathrm{T}_{p}$ as the discrete torus $\frac{1}{p} \mathbb{Z} / \mathbb{Z}$. Denote by $L^{2}\left(\mathrm{~T}_{p}\right)$ the $p$ complex dimensional Hilbert space of functions on $\mathrm{T}_{p}$ with the usual inner product $(\psi, \phi)=\sum_{\tau \in \mathrm{T}_{p}} \psi_{\tau}^{*} \phi_{\tau}$. Define the operator $\partial_{p}$ on $L^{2}\left(\mathrm{~T}_{p}\right)$ by

$$
\left(\partial_{p} \phi\right)_{\tau}=p\left(\phi_{\tau+1 / p}-\phi_{\tau}\right)
$$


Set, for each $0 \leq n \leq p-1$

$$
e_{n}(\tau)=e^{2 n \pi \imath \tau}
$$

Then $\left\{e_{n}(\tau)\right\}_{n=0, \cdots, p-1}$ is an (orthogonal) basis for $L^{2}\left(\mathrm{~T}_{p}\right)$ and each $e_{n}(\tau)$ is an eigenvector for any translation invariant operator on $L^{2}\left(\mathrm{~T}_{p}\right)$. Since

$$
\partial_{p} e_{n}(\tau)=p\left(e^{2 \pi \frac{n}{p} \imath}-1\right) e_{n}(\tau)
$$

the eigenvalues of $\partial_{p}$ are

$$
\lambda_{n, p}=p\left(e^{2 \pi \frac{n}{p} \imath}-1\right), \quad n=0,1, \cdots, p-1
$$

The $2 p$ real dimensional Gaussian integral

$$
N_{p}=\int \prod_{\tau \in \mathrm{T}_{p}} \frac{d \phi_{\tau}^{*} d \phi_{\tau}}{2 \pi i} e^{\frac{1}{p}\left(\phi,\left(\partial_{p}+\mu\right) \phi\right)}=\prod_{n=0}^{p-1}\left[-\frac{1}{p}\left(\lambda_{n, p}+\mu\right)\right]^{-1}
$$

We have

$$
\lim _{p \rightarrow \infty} \prod_{n=0}^{p-1}\left[-\frac{1}{p}\left(\lambda_{n, p}+\mu\right)\right]=\lim _{p \rightarrow \infty} \prod_{n=0}^{p-1}\left[1-\frac{\mu}{p}-e^{2 \pi \frac{n}{p}}\right]=\lim _{p \rightarrow \infty}\left(1-\frac{\mu}{p}\right)^{p}-1=e^{-\mu}-1
$$

since

$$
\prod_{n=0}^{p-1}\left(z-e^{\imath \frac{2 \pi n}{p}}\right)=z^{p}-1
$$

On the other hand, assuming that $p$ is divisible by 8 ,

$$
\int\left|d \nu_{p}\left(\phi^{*}, \phi\right)\right|=\int \prod_{\tau \in \mathrm{T}_{p}} \frac{d \phi_{\tau}^{*} d \phi_{\tau}}{2 \pi i}\left|e^{\frac{1}{p}\left(\phi,\left(\partial_{p}+\mu\right) \phi\right)}\right|=\prod_{n=0}^{p-1}\left[-\frac{1}{p} \operatorname{Re}\left(\lambda_{n, p}+\mu\right)\right]^{-1}
$$

and

$$
\begin{aligned}
& \prod_{n=0}^{p-1}\left[-\frac{1}{p} \operatorname{Re}\left(\lambda_{n, p}+\mu\right)\right]=\prod_{n=0}^{p-1}\left[C_{p}-\cos \left(2 \pi \frac{n}{p}\right)\right] \quad \text { where } C_{p}=1-\frac{\mu}{p} \\
& =\left(C_{p}-1\right)\left(C_{p}+1\right) \prod_{n=1}^{\frac{p}{2}-1}\left[C_{p}-\cos \left(2 \pi \frac{n}{p}\right)\right]^{2} \quad \text { since } \cos \left(2 \pi \frac{p-n}{p}\right)=\cos \left(2 \pi \frac{n}{p}\right) \\
& =\left(C_{p}-1\right) C_{p}^{2}\left(C_{p}+1\right) \prod_{n=1}^{\frac{p}{4}-1}\left[C_{p}^{2}-\cos ^{2}\left(2 \pi \frac{n}{p}\right)\right]^{2} \quad \text { since } \cos \left(2 \pi \frac{p / 2-n}{p}\right)=-\cos \left(2 \pi \frac{n}{p}\right) \\
& =\left(C_{p}-1\right) C_{p}^{2}\left(C_{p}+1\right)\left(C_{p}^{2}-\frac{1}{2}\right)^{2} \prod_{n=1}^{\frac{p}{8}-1}\left[C_{p}^{2}-\cos ^{2}\left(2 \pi \frac{n}{p}\right)\right]^{2}\left[C_{p}^{2}-\sin ^{2}\left(2 \pi \frac{n}{p}\right)\right]^{2} \\
& \text { since } \cos \left(2 \pi \frac{p / 4-n}{p}\right)=\sin \left(2 \pi \frac{n}{p}\right)
\end{aligned}
$$


Now

$$
\begin{aligned}
{\left[C_{p}^{2}-\cos ^{2}\left(2 \pi \frac{n}{p}\right)\right]^{2}\left[C_{p}^{2}-\sin ^{2}\left(2 \pi \frac{n}{p}\right)\right]^{2} } & =\left[C_{p}^{4}-C_{p}^{2}+\sin ^{2}\left(2 \pi \frac{n}{p}\right) \cos ^{2}\left(2 \pi \frac{n}{p}\right)\right]^{2} \\
& =\left[C_{p}^{2}\left(-2 \frac{\mu}{p}+\frac{\mu^{2}}{p^{2}}\right)+\frac{1}{4} \sin ^{2}\left(4 \pi \frac{n}{p}\right)\right]^{2} \\
& \leq \frac{1}{10}
\end{aligned}
$$

if $p$ is large enough, since $\lim _{p \rightarrow \infty} C_{p}=1$ and $\lim _{p \rightarrow \infty}\left(-2 \frac{\mu}{p}+\frac{\mu^{2}}{p^{2}}\right)=0$. If $p$ is large enough, we also have

$$
\left(C_{p}-1\right) C_{p}^{2}\left(C_{p}+1\right)\left(C_{p}^{2}-\frac{1}{2}\right)^{2}=-\frac{\mu}{p} C_{p}^{2}\left(C_{p}+1\right)\left(C_{p}^{2}-\frac{1}{2}\right)^{2}<\frac{1}{10}
$$




\section{References}

[B] T. Balaban, A Low Temperature Expansion and "Spin Wave Picture" for Classical $N$-Vector Models, in Constructive Physics, V. Rivasseau, editor, Springer, 1995.

[C] R. Cameron, The Ilstow and Feynman Integrals, J. Analyse Math 10 (1962/63), $287-361$.

[FSS] J. Fröhlich, B. Simon and T. Spencer, Infrared Bounds, Phase Transitions and Continuous Symmetry Breaking, Commun. Math. Phys. 50, 79-95 (1976).

[GV] I. M. Gel'fand and N. Ya. Vilenkin, Generalized functions. Applications of harmonic analysis, Vol 4 , Acad. Press (1968).

[II] T. Balaban, J. Feldman, H. Knörrer and E. Trubowitz, A Functional Integral Representation for Many Boson Systems. II: Correlation Functions, preprint.

[NO] John W. Negele and Henri Orland, Quantum Many-Particle Systems, AddisonWesley (1988). 